\title{
Three-dimensional coating and rimming flow: a ring of fluid on a rotating horizontal cylinder
}

\author{
G. A. LESLIE，S.K. WILSON $\dagger$ AND B. R. DUFF Y \\ Department of Mathematics and Statistics, University of Strathclyde, 26 Richmond Street, \\ Glasgow G1 1XH, UK \\ (Received ?; revised ?; accepted ?.)
}

The steady three-dimensional flow of a thin, slowly varying ring of Newtonian fluid on either the outside or the inside of a uniformly rotating large horizontal cylinder is investigated. Specifically, we study "full-ring" solutions, corresponding to a ring of continuous, finite and non-zero thickness that extends all the way around the cylinder. In particular, it is found that there is a critical solution corresponding to either a critical load above which no full-ring solution exists (if the rotation speed is prescribed) or a critical rotation speed below which no full-ring solution exists (if the load is prescribed). We describe the behaviour of the critical solution and, in particular, show that the critical flux, the critical load, the critical semi-width and the critical ring profile are all increasing functions of the rotation speed. In the limit of small rotation speed, the critical flux is small and the critical ring is narrow and thin, leading to a small critical load. In the limit of large rotation speed, the critical flux is large and the critical ring is wide on the upper half of the cylinder and thick on the lower half of the cylinder, leading to a large critical load. We also describe the behaviour of the non-critical full-ring solution, and, in particular, show that the semi-width and the ring profile are increasing functions of the load but, in general, non-monotonic functions of the rotation speed. In the limit of large rotation speed, the ring approaches a limiting non-uniform shape, whereas in the limit of small load, the ring is narrow and thin with a uniform parabolic profile. Finally, we show that, while for most values of the rotation speed and the load the azimuthal velocity is in the same direction as the rotation of the cylinder, there is a region of parameter space close to the critical solution for sufficiently small rotation speed in which backflow occurs in a small region on the upward-moving side of the cylinder.

\section{Introduction}

Even before the pioneering work by Pukhnachev (1977) and Moffatt (1977) there was theoretical and experimental interest in flows on rotating horizontal cylinders, but since the publication of these seminal works there has been considerable and ongoing theoretical, numerical and experimental research activity on both "coating flow" (i.e. flow on the outside surface) and "rimming flow" (i.e. flow on the inside surface) on a uniformly rotating horizontal cylinder.

$\dagger$ Author for correspondence. Presently also a Visiting Fellow in the Oxford Centre for Collaborative Applied Mathematics (OCCAM), University of Oxford, Mathematical Institute, 24-29 St. Giles', Oxford OX1 3LB. Email: s.k.wilson@strath.ac.uk

$\ddagger$ Email: b.r.duffy@strath.ac.uk

ब This author's name has been transliterated into English as both "Pukhnachev" and "Pukhnachov". 
Both coating flow and, more especially, rimming flow have proved to be a fascinating and fruitful "test bed" for the study of a wide range of fluid-dynamical phenomena, including (in no particular order) fluid instabilities, solitary waves, pattern formation, multiple steady and unsteady states (including chaotic behavior), segregation in dilute suspensions, and avalanches in granular materials. As a result there is a considerable and steadily growing literature on both coating and rimming flows. Following the work by Pukhnachev (1977) and Moffatt (1977), important contributions have been made by many authors, including Johnson (1988), Preziosi \& Joseph (1988), Melo \& Douady (1993), Thoroddsen \& Mahadevan (1997), Hosoi \& Mahadevan (1999), Duffy \& Wilson (1999), Peterson et al. (2001), Wilson et al. (2002b), Ashmore et al. (2003), Hinch \& Kelmanson (2003), Acrivos \& Jin (2004), Evans et al. (2004), Benilov \& O'Brien (2005), Evans et al. (2005), Villegas-Díaz et al. (2005), Benilov (2006), Noakes et al. (2006), Chen et al. (2007), Benilov et al. (2008), Hunt (2008), Benilov et al. (2009), Kelmanson (2009), Shrager et al. (2009), Tougher et al. (2009), Chicharro et al. (2011), Pougatch \& Frigaard (2011), Thiele (2011), Benilov et al. (2012), Leslie et al. (2012), and Williams et al. (2012). The recent review article by Seiden \& Thomas (2011) focuses on pattern formation and segregation in rimming flows of dilute suspensions and granular materials, but also provides probably the most complete overview of the literature on coating and rimming flows of pure fluids currently available.

Inevitably most of the theoretical and numerical work has focused on the most tractable cases of two-dimensional or weakly three-dimensional flows. In particular, Moffatt (1977) found that steady two-dimensional solutions corresponding to a thin film of fluid covering the entire cylinder are possible only below a critical maximum load, and that, rather counter intuitively, these solutions always have top-to-bottom symmetry (i.e. they are the same on the top and the bottom halves of the cylinder).

The present work provides the first theoretical analysis of a three-dimensional coating and rimming flow consisting of "rings" (sometimes also referred to as "bands" or "strips") of fluid flowing around a uniformly rotating horizontal cylinder. As we shall see, not only is this flow of considerable interest in its own right, but may also be relevant to the complex three-dimensional flows observed experimentally by, for example, Moffatt (1977), Melo \& Douady (1993), Thoroddsen \& Mahadevan (1997) and Chen et al. (2007) as well as the banded films of condensed ammonia-water mixtures observed experimentally by Deans \& Kucuka (2011) on the outer surface of a stationary horizontal cylinder.

Although, on account of their inherent three-dimensionality, there has been relatively little previous theoretical work on rings, there is a large literature on the related problem of rivulet flow. In particular, Towell \& Rothfeld (1966) and Allen \& Biggin (1974) studied the steady unidirectional flow of a uniform rivulet (i.e. a rivulet with constant crosssectional profile) of Newtonian fluid down an inclined plane, and subsequently Duffy \& Moffatt (1995) obtained the solution for a thin rivulet and interpreted their results as describing the locally unidirectional flow of a thin rivulet down a slowly varying substrate, and, in particular, as describing the flow in the azimuthal direction around a large horizontal cylinder. There has been considerable work on many different aspects of rivulet flow; in the absence of a recent review article, the work by Schmuki \& Laso (1990), Duffy \& Moffatt (1997), Wilson \& Duffy (1998), Roy \& Schwartz (1999), Wilson et al. (2002a), Kim et al. (2004), Perazzo \& Gratton (2004), Saber \& El-Genk (2004), Wilson \& Duffy (2005), Le Grand-Piteira et al. (2006), Alekseenko et al. (2008), Sullivan et al. (2008), Benilov (2009), Diez et al. (2009), Tanasijczuk et al. (2010), Daerr et al. (2011), Wilson et al. (2011) and Yatim et al. (2011) (and the references therein) provide a representative selection of the recent literature.

In the present paper we use a combination of asymptotic and numerical methods to 
investigate the steady three-dimensional flow of a thin, slowly varying ring of Newtonian fluid on either the outside or the inside of a uniformly rotating large horizontal cylinder. Specifically, we study what we will call "full-ring" solutions, corresponding to a ring of continuous, finite and non-zero thickness that extends all the way around the cylinder. This study therefore combines aspects of the classical two-dimensional coating and rimming flow studied by Moffatt (1977) (such as, for example, the existence of a critical maximum load for the fluid to extend all the way around the cylinder) with aspects of the locally unidirectional flow of a thin rivulet down a slowly varying substrate studied by Duffy \& Moffatt (1995) (such as, for example, the non-uniform profile and the lack of top-to-bottom symmetry) to provide the first theoretical analysis of this interesting and previously largely unexplored three-dimensional flow.

\section{Problem formulation}

Consider the steady three-dimensional flow of a thin, slowly varying ring of Newtonian fluid with uniform density $\rho$ and viscosity $\mu$ on either the outside or the inside of a large circular cylinder of radius $R$ rotating in a counter-clockwise direction about its horizontal axis at uniform angular speed $\Omega(>0)$ (so that the circumferential speed, denoted by $U$, is $U=R \Omega$ ). Referred to polar coordinates $\theta$ (the azimuthal coordinate measured counter-clockwise from the horizontal), $Y$ (the axial coordinate with origin at the axis of symmetry of the ring) and $r=R \pm Z$ (the radial coordinate with origin at the cylinder's axis), as shown in figure 1 (drawn for the case of flow on the outside of the cylinder), we take the free surface of the ring to be at $r=R+h$ for flow on the outside of the cylinder and $r=R-h$ for flow on the inside of the cylinder, the ring profile being denoted by $h=h(\theta, Y)$, and take the contact lines at the edges of the ring (where $h=0$ ) to be at $Y= \pm a$, the semi-width being denoted by $a=a(\theta)(>0)$. Hence the fluid lies in the intervals $-a \leqslant Y \leqslant a$ and $0 \leqslant Z \leqslant h$ for $-\pi<\theta \leqslant \pi$. The fluid velocity $\mathbf{u}=u \mathbf{e}_{\theta}+v \mathbf{e}_{Y}+w \mathbf{e}_{r}$ (where $\mathbf{e}_{\theta}, \mathbf{e}_{Y}$ and $\mathbf{e}_{r}$ denote unit vectors in the azimuthal, axial and radial directions, respectively) and pressure $p$ are governed by the usual massconservation and Navier-Stokes equations. On the cylinder $r=R$ the velocity $\mathbf{u}$ satisfies no-slip and no-penetration conditions, while on the free surface $r=R \pm h$ the usual normal and tangential stress balances and the kinematic condition apply.

We consider a thin, slowly varying ring whose longitudinal aspect ratio, denoted $\epsilon$, is defined to be $\epsilon=\ell / R \ll 1$, where $\ell=(\gamma / \rho g)^{1 / 2}$ is the capillary length in which $\gamma$ is the constant coefficient of surface tension and $g$ is the magnitude of gravitational acceleration, and whose transverse aspect ratio is defined to be the prescribed contact angle, denoted by $\beta \ll 1$. In particular, we consider the situation in which the ring is sufficiently slowly varying (i.e. the cylinder is sufficiently large) that $\epsilon \ll \beta \ll 1$. Hence we non-dimensionalise and scale the system appropriately by writing

$$
\left.\begin{array}{c}
r=R\left(1 \pm \epsilon \beta Z^{*}\right), \quad Y=\epsilon R Y^{*}, \quad \theta= \pm \theta^{*}, \quad h=\epsilon \beta R h^{*}, \quad a=\epsilon R a^{*}, \\
p-p_{\mathrm{a}}=\epsilon \beta R \rho g p^{*}, \quad u=\frac{\epsilon^{2} \beta^{2} R^{2} \rho g}{\mu} u^{*}, \quad v= \pm \frac{\epsilon^{3} \beta^{2} R^{2} \rho g}{\mu} v^{*}, \quad w=\frac{\epsilon^{3} \beta^{3} R^{2} \rho g}{\mu} w^{*}, \\
Q=\frac{\epsilon^{4} \beta^{3} R^{4} \rho g}{\mu} Q^{*}, \quad M=\epsilon^{2} \beta R^{3} \rho M^{*}, \quad U=R \Omega=\frac{\epsilon^{2} \beta^{2} R^{2} \rho g}{\mu} U^{*},
\end{array}\right\}
$$

in which the $+\operatorname{sign}(-\operatorname{sign})$ corresponds to flow on the outside (inside) of the cylinder, $p_{\mathrm{a}}$ is the constant pressure in the surrounding atmosphere, $Q$ is the azimuthal volume flux of fluid crossing a station $\theta=$ constant, and $M(>0)$ is the constant fluid load (i.e. the mass of fluid) on the cylinder. For clarity the star superscripts on the non-dimensional 


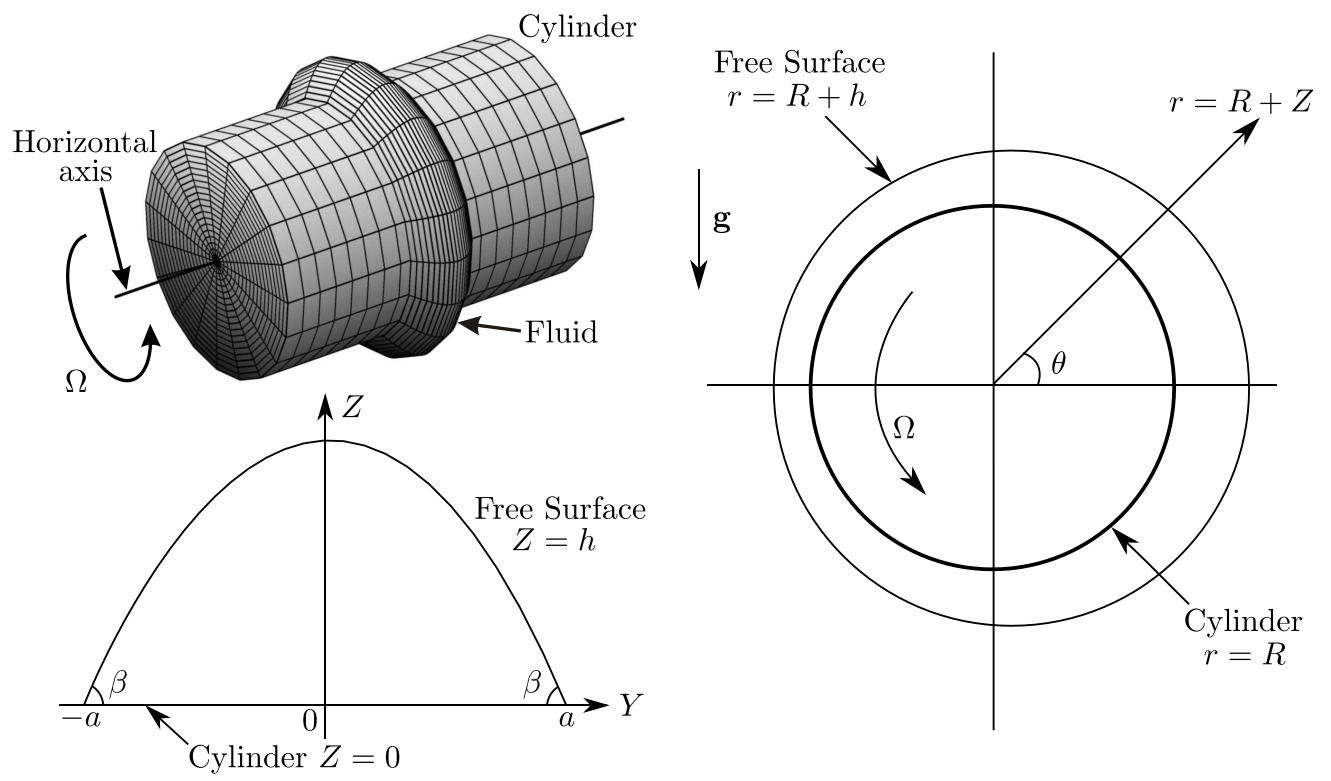

Figure 1. Geometry of the problem: steady three-dimensional flow of a thin, slowly varying ring of Newtonian fluid on a uniformly rotating horizontal cylinder.

quantities will be omitted henceforth. We shall describe what follows in terms of flow on the outside of the cylinder only (i.e. we choose the + in the \pm above) so that, in particular, positive values of $\theta$ correspond to the top of the cylinder and negative values to the bottom; to interpret the results that follow for flow on the inside of the cylinder it is simply necessary to let positive values of $\theta$ correspond to the bottom of the cylinder and negative values to the top. (Note that this swapping of top and bottom when switching between coating and rimming flow also occurs in the corresponding two-dimensional problem.)

At leading order in $\epsilon$ and $\beta$ the governing equations for the flow on the cylinder become

$$
\frac{\partial u}{\partial \theta}+\frac{\partial v}{\partial Y}+\frac{\partial w}{\partial Z}=0, \quad \frac{\partial^{2} u}{\partial Z^{2}}=\cos \theta, \quad \frac{\partial p}{\partial Y}=0, \quad \frac{\partial p}{\partial Z}=-\sin \theta
$$

together with the boundary conditions

$$
\begin{gathered}
u=U, \quad v=0 \quad \text { and } \quad w=0 \quad \text { on } \quad Z=0, \\
p=-\frac{\partial^{2} h}{\partial Y^{2}}, \quad \frac{\partial u}{\partial Z}=0 \quad \text { and } \quad \frac{\partial v}{\partial Z}=0 \quad \text { on } \quad Z=h,
\end{gathered}
$$

the kinematic condition on $Z=h$, which may be written in the form

$$
\frac{\partial \bar{u}}{\partial \theta}+\frac{\partial \bar{v}}{\partial Y}=0
$$

where the local azimuthal and axial fluxes, $\bar{u}=\bar{u}(\theta, Y)$ and $\bar{v}=\bar{v}(\theta, Y)$, are defined by

$$
\bar{u}=\int_{0}^{h} u \mathrm{~d} Z, \quad \bar{v}=\int_{0}^{h} v \mathrm{~d} Z,
$$

and

$$
h=0 \quad \text { and } \quad \frac{\partial h}{\partial Y}=\mp 1 \quad \text { on } \quad Y= \pm a .
$$


Introducing the rescaled axial coordinate $y=Y / a$ and rescaled radial coordinate $z=$ $Z / h$ (so that the fluid lies within the fixed intervals $-1 \leqslant y \leqslant 1$ and $0 \leqslant z \leqslant 1$ for $-\pi<\theta \leqslant \pi)$ and integrating $(2.2)_{4}$ subject to $(2.4)_{1}$ gives the solution for the pressure:

$$
p=h \sin \theta(1-z)-\frac{1}{a^{2}} \frac{\partial^{2} h}{\partial y^{2}},
$$

from which $(2.2)_{3}$ gives a third-order ordinary differential equation for the ring profile $h=h(\theta, y)$, namely

$$
\frac{\partial}{\partial y}\left(h \sin \theta-\frac{1}{a^{2}} \frac{\partial^{2} h}{\partial y^{2}}\right)=0
$$

whose solution satisfying $(2.7)$ is

$$
h= \begin{cases}\frac{\cosh m a-\cosh m a y}{m \sinh m a} & \text { if } 0<\theta<\pi, \\ \frac{a\left(1-y^{2}\right)}{2} & \text { if } \theta=0 \text { or } \theta=\pi, \\ \frac{\cos m a y-\cos m a}{m \sin m a} & \text { if }-\pi<\theta<0,\end{cases}
$$

where for convenience we have introduced the notation $m=|\sin \theta|^{1 / 2}$. At any station $\theta=$ constant the ring profile $h$ given by (2.10) is symmetric about $y=0$, with a single maximum at $y=0$, and the maximum thickness of the ring, denoted $h_{\mathrm{m}}=h_{\mathrm{m}}(\theta)=$ $h(\theta, 0)$, is given by

$$
h_{\mathrm{m}}= \begin{cases}\frac{1}{m} \tanh \left(\frac{m a}{2}\right) & \text { if } 0<\theta<\pi, \\ \frac{a}{2} & \text { if } \theta=0 \text { or } \theta=\pi, \\ \frac{1}{m} \tan \left(\frac{m a}{2}\right) & \text { if }-\pi<\theta<0 .\end{cases}
$$

Note that the local solution for the profile of the ring is equivalent to the profile of a thin rivulet flowing down a plane inclined at an angle $\pi / 2-\theta$ to the horizontal given by Duffy \& Moffatt (1995). Physically the flow is a balance between gravity and viscous forces, with the cross-sectional profile determined by a balance between surface-tension and gravity forces. For future reference it is useful to note that $h$ is given by

$$
h=\frac{a}{2}\left(1-y^{2}\right)-\operatorname{sgn}(\theta) \frac{m^{2} a^{3}}{24}\left(1-y^{2}\right)^{2}+O\left(m^{4} a^{5}\right)
$$

in the limits $a \rightarrow 0^{+}$and $m \rightarrow 0^{+}$, while for $0<\theta<\pi$ it is given by

$$
h=\frac{1}{m}(1-2 \exp (-m a) \cosh m a y)+O(\exp (-2 m a))
$$

in the limit $a \rightarrow \infty$, and for $-\pi<\theta<0$ it is given by

$$
h=\frac{1+\cos \pi y}{m(\pi-m a)}+\frac{y \sin \pi y}{m}+O(\pi-m a)
$$

in the limit $m a \rightarrow \pi$.

In the present work, we shall henceforth be concerned only with what we will call "full-ring" solutions, i.e. solutions for which $a$ and $h$ are continuous, finite and non-zero for all $-\pi<\theta \leqslant \pi$ and $-1<y<1$, corresponding to a continuous ring of finite, nonzero width and thickness that extends all the way around the cylinder. These full-ring 
solutions may be thought of as a three-dimensional generalisation of the two-dimensional "full-film" solutions described by Moffatt (1977), and, like them, have a constant (but as yet unknown) volume flux $Q$. Note, however, that the full-ring solutions do not, in general, have the rather counter-intuitive top-to-bottom symmetry exhibited by the fullfilm solutions. Integrating $(2.2)_{2}$ twice subject to $(2.3)_{1}$ and $(2.4)_{2}$ gives the azimuthal velocity

$$
u=U-\frac{h^{2} \cos \theta}{2}(2-z) z,
$$

and so the local azimuthal flux $\bar{u}$ is given by

$$
\bar{u}=U h-\frac{h^{3} \cos \theta}{3},
$$

and hence the constant flux $Q$ is given by

$$
Q=a \int_{-1}^{1} \bar{u} \mathrm{~d} y=U a \int_{-1}^{1} h \mathrm{~d} y-\frac{a \cos \theta}{3} \int_{-1}^{1} h^{3} \mathrm{~d} y
$$

which leads to

$$
Q=-\frac{\cos \theta}{9 m^{4}} F(m a)+\frac{U}{m^{2}} G(m a)
$$

where the functions $F(m a)$ and $G(m a)$ are defined by

$$
F(m a)= \begin{cases}15 m a \operatorname{coth}^{3} m a-15 \operatorname{coth}^{2} m a-9 m a \operatorname{coth} m a+4 & \text { if } 0<\theta<\pi, \\ -15 m a \cot ^{3} m a+15 \cot ^{2} m a-9 m a \cot m a+4 & \text { if }-\pi<\theta<0\end{cases}
$$

and

$$
G(m a)= \begin{cases}2(m a \operatorname{coth} m a-1) & \text { if } 0<\theta<\pi, \\ -2(m a \cot m a-1) & \text { if }-\pi<\theta<0,\end{cases}
$$

and appropriate interpretation of the special cases $\theta=0$ and $\theta=\pi$ as limits is required. The functions $F(m a)$ and $G(m a)$ are plotted together with their derivatives $F^{\prime}(m a)$ and $G^{\prime}(m a)$ in figure 2. For $0<\theta<\pi$ the functions $F(m a)$ and $G(m a)$ are positive functions of $m a$, increasing monotonically from zero at $m a=0$ to infinity with finite slope as $m a \rightarrow \infty$, whereas for $-\pi<\theta<0$ the functions $F(m a)$ and $G(m a)$ have multiple branches; however, one may show that the branch that gives rise to full-ring solutions lies in the interval $0<m a<\pi$ within which they are positive functions of $m a$, increasing monotonically from zero at $m a=0$ to infinity with infinite slope as $m a \rightarrow \pi$. For future reference it is useful to note that $F(m a)$ and $G(m a)$ are given by

$$
F(m a)=\frac{12}{35}(m a)^{4}-\operatorname{sgn}(\theta) \frac{8}{105}(m a)^{6}+O\left((m a)^{8}\right)
$$

and

$$
G(m a)=\frac{2}{3}(m a)^{2}-\operatorname{sgn}(\theta) \frac{2}{45}(m a)^{4}+O\left((m a)^{6}\right)
$$

in the limit $m a \rightarrow 0$, while for $0<\theta<\pi$ they are given by

$$
F(m a)=6 m a-11+12(6 m a-5) \exp (-2 m a)+O(m a \exp (-4 m a))
$$

and

$$
G(m a)=2 m a-2+4 m a \exp (-2 m a)+O(m a \exp (-4 m a))
$$



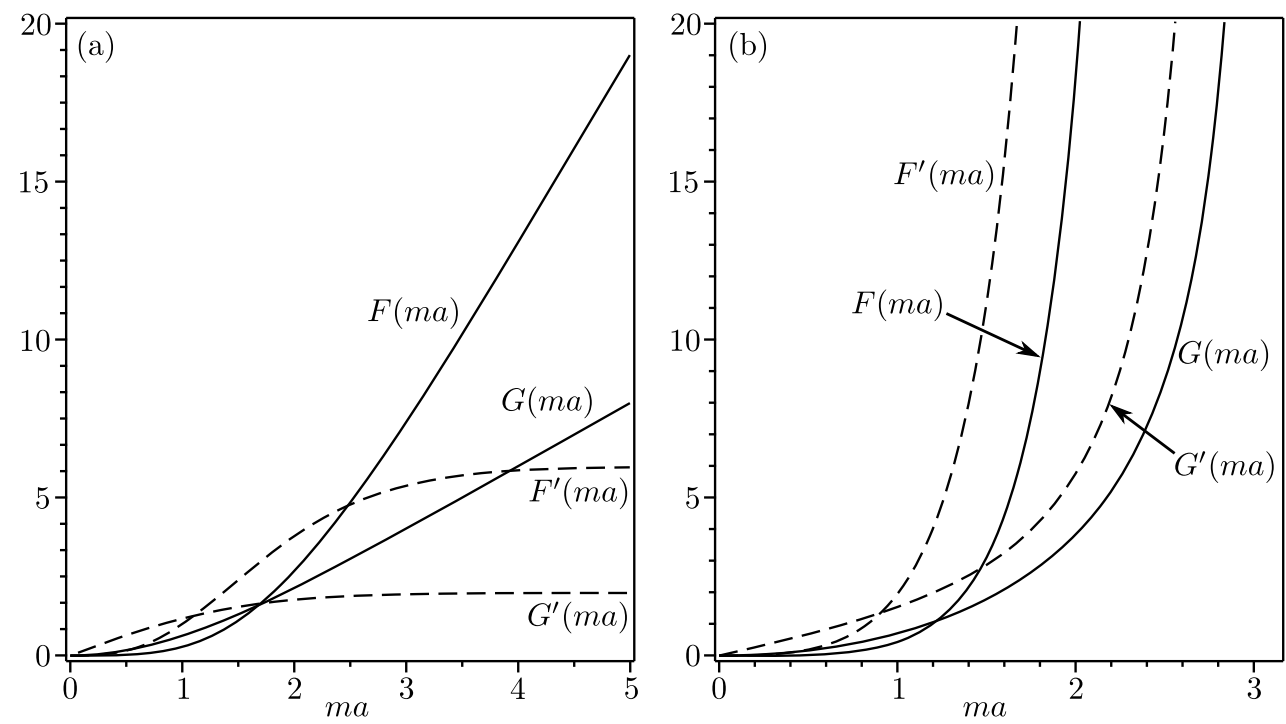

Figure 2. The functions $F(m a)$ and $G(m a)$ (solid lines), defined by (2.19) and (2.20), respectively, together with their derivatives $F^{\prime}(m a)$ and $G^{\prime}(m a)$ (dashed lines) for (a) $0<\theta<\pi$ and (b) $-\pi<\theta<0$ in the interval $0<m a<\pi$.

in the limit $m a \rightarrow \infty$, and for $-\pi<\theta<0$ they are given by

$$
F(m a)=\frac{15 \pi}{(\pi-m a)^{3}}-\frac{6 \pi}{\pi-m a}+O(\pi-m a)
$$

and

$$
G(m a)=\frac{2 \pi}{\pi-m a}-\frac{2 \pi}{3}(\pi-m a)+O\left((\pi-m a)^{2}\right)
$$

in the limit $m a \rightarrow \pi$.

Equation (2.18) provides an implicit solution for the semi-width $a=a(\theta)$ in terms of the flux $Q$. At the top and bottom of the cylinder (i.e. at $\theta= \pm \pi / 2$ ) the flux $Q$ given by (2.18) takes the form $Q=U G(a)(>0)$, while at $\theta=0$ and $\theta=\pi$ it yields $Q=-4 a^{4} / 105+2 U a^{2} / 3$ and $Q=4 a^{4} / 105+2 U a^{2} / 3$, respectively. While the roots of the former quadratic for $a^{2}$ are always real, those of the latter are only real provided that $0<Q \leqslant 35 U^{2} / 12$, showing that for full-ring solutions $Q$ must always lie in this interval.

Figure 3 shows three contours of the expression for the flux $Q$ given by (2.18) in the $\theta / \pi-a$ plane when $U=1$ (contours for other values of $U$ are qualitatively similar) which, since they are by definition curves on which $Q=$ constant, represent candidate solutions for the semi-width $a=a(\theta)$. This figure clearly demonstrates one of the key features of the present problem, namely the existence of a critical solution with a critical flux, denoted by $Q=Q_{\mathrm{c}}$, such that full-ring solutions exist when $Q \leqslant Q_{\mathrm{c}}$ but not when $Q>Q_{\mathrm{c}}$. Here and henceforth variables in the critical case are denoted with a subscript c, e.g. $Q_{\mathrm{c}}$. This solution, which is analogous to the critical two-dimensional full-film solution described by Moffatt (1977), is discussed in Section 3. In particular, figure 3(a) shows that when $Q<Q_{\text {c }}$ only the lowest branch (shown with a solid line) is a full-ring solution, while the higher branches (shown with dashed lines) form a rather complicated "network" which does not extend over all $-\pi<\theta \leqslant \pi$ and so cannot be a full-ring solution. Figure 3(b) shows that when $Q=Q_{\mathrm{c}}$ the critical full-ring solution is made up of two different branches (again shown with a solid line) which meet to form a corner at $\theta=\hat{\theta}_{\mathrm{c}}$ and $a=\hat{a}_{\mathrm{c}}$, while 

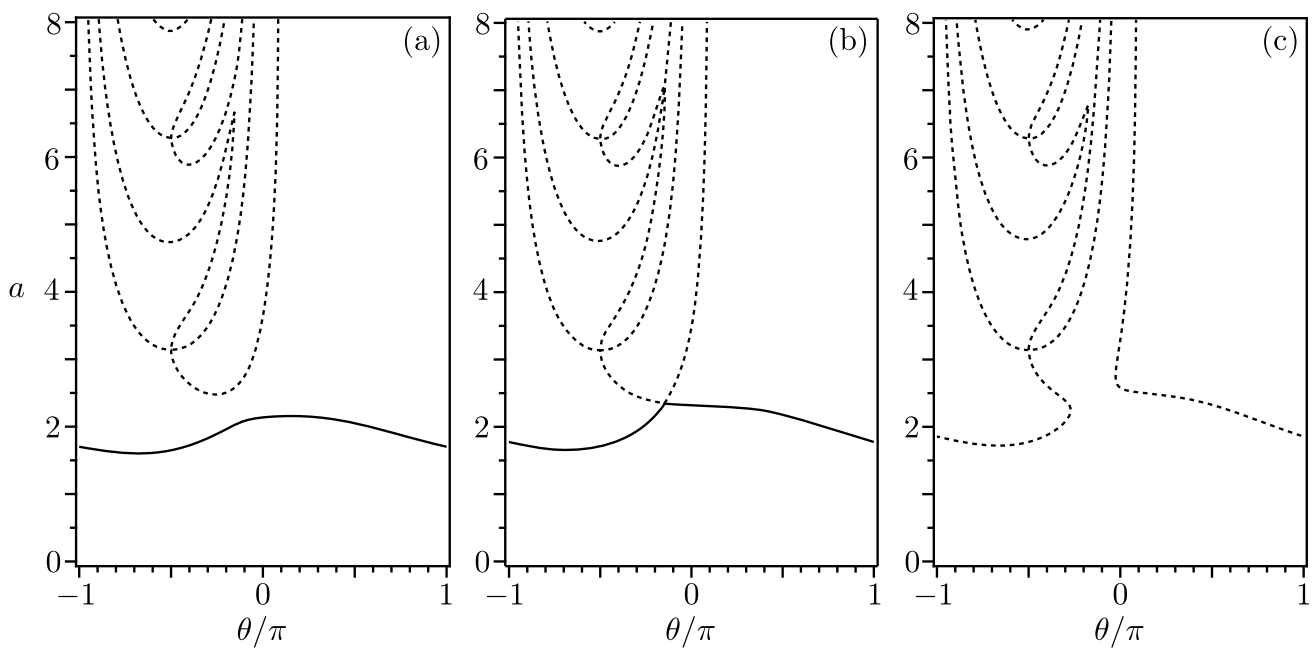

Figure 3. Three contours of the expression for the flux $Q$ given by (2.18) in the $\theta / \pi-a$ plane when $U=1$. The contours are drawn for (a) $Q=2.25\left(<Q_{\mathrm{c}}=10 \sqrt{5} / 9 \simeq 2.48452\right)$, (b) $Q=Q_{\mathrm{c}}$ and (c) $Q=2.75\left(>Q_{\mathrm{c}}\right)$. The branches corresponding to full-ring solutions are shown with solid lines, while the other branches are shown with dashed lines.

again the higher branches (again shown with dashed lines) cannot be a full-ring solution. Here and henceforth variables relating to the corner in the critical case are denoted with both a subscript c and a hat, e.g. $\hat{\theta}_{\mathrm{c}}$. Figure $3(\mathrm{c})$ shows that when $Q>Q_{\mathrm{c}}$ there is no branch that extends over all $-\pi<\theta \leqslant \pi$ and so no full-ring solution exists. Of course, as, for example, Johnson (1988) describes in the two-dimensional case, as well as the continuous full-ring solutions there can also be piecewise-continuous "shock" or "jump" solutions with one or more discontinuities at which the solution jumps between different branches. In particular, figure 3(b) shows that, as in the two-dimensional case, in the critical case there can be a solution with only one discontinuity. Piecewise-continuous solutions are not considered further in the present work, but are an interesting topic for future work.

The total fluid load on the cylinder, $M$, is given by

$$
M=\int_{-\pi}^{\pi} \int_{-1}^{1} \int_{0}^{1} a(\theta) h(\theta, y) \mathrm{d} z \mathrm{~d} y \mathrm{~d} \theta
$$

leading to

$$
M=\int_{-\pi}^{\pi} \frac{G(m a)}{m^{2}} \mathrm{~d} \theta
$$

where the function $G(m a)$ is again given by $(2.20)$.

With the rotation speed $U$ prescribed, the semi-width $a$, and hence the ring profile $h$ given by (2.10), is determined in terms of $Q$ by the algebraic equation (2.18) in which the functions $F(m a)$ and $G(m a)$ are given by (2.19) and (2.20), respectively. The constant but unknown value of $Q$ is determined either from an appropriate criticality condition or from the condition of prescribed load using (2.28). The properties and behaviour of the solutions in these two cases are discussed in Sections 3 and 4, respectively, but in both cases, the solutions for $p, h$ and $u$ are given explicitly by (2.8), (2.10) and (2.15), respectively. If required, $v$ can (in principle) be determined by proceeding to higher order in $(2.2)_{3}$ and then $w$ can be determined from the leading order mass-conservation equation $(2.2)_{1}$. 


\section{The critical full-ring solution}

In this Section we describe the behaviour of the critical full-ring solution with critical flux $Q=Q_{\mathrm{c}}$. Not only is this solution of considerable interest in its own right but, as we shall see, investigating this critical case is necessary in order to understand when a non-critical full-ring solution exists when both the rotation speed $U$ and the load $M$ are prescribed (this solution is discussed in Section 4). In the critical case either $U$ or $M$ may be prescribed with the other being determined by the criticality condition; specifically, as we shall see, if the rotation speed $U$ is prescribed then there is a critical load, denoted by $M=M_{\mathrm{c}}$, above which no full-ring solutions are possible, whereas if the load $M$ is prescribed then there is a critical rotation speed, denoted by $U=U_{\mathrm{c}}$, below which no fullring solutions are possible. For simplicity and to avoid repetition we present the following analysis for the case of prescribed rotation speed $U$ and leave the reader to interpret the results appropriately for the case of prescribed load $M$.

As may be seen from figure 3(b), a key feature of the critical solution is that the expression for the flux $Q$ given by (2.18) has a saddle point at $\theta=\hat{\theta}_{\mathrm{c}}$ and $a=\hat{a}_{\mathrm{c}}$ which gives rise to a corner in the critical full-ring profile $h_{\mathrm{c}}$ at $\theta=\hat{\theta}_{\mathrm{c}}$. This corner is analogous to the corner in the critical two-dimensional full-film profile described by Moffatt (1977), except, of course, that it occurs on a curve in three dimensions (rather than at a point in two dimensions). At the saddle point we have the necessary (but not sufficient) conditions $\partial Q / \partial \theta=0$ and $\partial Q / \partial a=0$, which, from (2.18), leads to the criticality conditions

$$
\left(1+\cos ^{2} \hat{\theta}_{\mathrm{c}}\right) F\left(\hat{m}_{\mathrm{c}} \hat{a}_{\mathrm{c}}\right)=9 U \cos \hat{\theta}_{\mathrm{c}}\left|\sin \hat{\theta}_{\mathrm{c}}\right| G\left(\hat{m}_{\mathrm{c}} \hat{a}_{\mathrm{c}}\right)
$$

and

$$
\cos \hat{\theta}_{\mathrm{c}} F^{\prime}\left(\hat{m}_{\mathrm{c}} \hat{a}_{\mathrm{c}}\right)=9 U\left|\sin \hat{\theta}_{\mathrm{c}}\right| G^{\prime}\left(\hat{m}_{\mathrm{c}} \hat{a}_{\mathrm{c}}\right),
$$

where we have defined $\hat{m}_{\mathrm{c}}=\left|\sin \hat{\theta}_{\mathrm{c}}\right|^{1 / 2}$. We may eliminate $\hat{\theta}_{\mathrm{c}}$ and $\hat{a}_{\mathrm{c}}$ from (3.1) and (3.2) in favour of $\hat{B}_{\mathrm{c}}=\hat{m}_{\mathrm{c}} \hat{a}_{\mathrm{c}}(>0)$ in order to obtain a single algebraic equation for $\hat{B}_{\mathrm{c}}$ involving the parameter $U$ only, namely

$$
\frac{F\left(\hat{B}_{\mathrm{c}}\right) F^{\prime}\left(\hat{B}_{\mathrm{c}}\right)^{2}}{G^{\prime}\left(\hat{B}_{\mathrm{c}}\right)\left[F^{\prime}\left(\hat{B}_{\mathrm{c}}\right) G\left(\hat{B}_{\mathrm{c}}\right)-2 F\left(\hat{B}_{\mathrm{c}}\right) G^{\prime}\left(\hat{B}_{\mathrm{c}}\right)\right]}=81 U^{2} .
$$

From the criticality condition (3.2) it is immediately apparent that $\cos \hat{\theta}_{c}>0$, and so we deduce that the corner always lies on the upward-moving side of the cylinder (i.e. in the interval $-\pi / 2<\hat{\theta}_{\mathrm{c}}<\pi / 2$ ). When $0<\hat{\theta}_{\mathrm{c}}<\pi / 2$ the function on the lefthand side of (3.3) is negative for all $\hat{B}_{\mathrm{c}}>0$, and so (3.3) has no full-ring solution for $\hat{B}_{\mathrm{c}}$ for any $U$; on the other hand, when $-\pi / 2<\hat{\theta}_{\mathrm{c}}<0$ this function is positive for $0<\hat{B}_{\mathrm{c}}<\pi$, increasing monotonically from zero at $\hat{B}_{\mathrm{c}}=0$ to infinity as $\hat{B}_{\mathrm{c}} \rightarrow \pi$, and so for any prescribed $U$ equation (3.3) has a unique full-ring solution for $\hat{B}_{\mathrm{c}}$. Then, with $\hat{B}_{\mathrm{c}}$ determined numerically from (3.3), the position of the corner $\hat{\theta}_{\mathrm{c}}=\hat{\theta}_{\mathrm{c}}(U)$ is given by $(3.2)$ as

$$
\hat{\theta}_{\mathrm{c}}=-\tan ^{-1}\left(\frac{F^{\prime}\left(\hat{B}_{\mathrm{c}}\right)}{9 U G^{\prime}\left(\hat{B}_{\mathrm{c}}\right)}\right),
$$

which together with (3.3) may be used to show that $-\pi / 4<\hat{\theta}_{\mathrm{c}}<0$. In particular, (3.4) shows that, unlike for the critical two-dimensional full-film solution described by Moffatt (1977) in which the corner is always at $\theta=0$, the position of the corner in the present critical full-ring solution varies with $U$. The values of the critical semi-width and critical maximum thickness at the corner, $\hat{a}_{\mathrm{c}}=\hat{a}_{\mathrm{c}}(U)=a\left(\hat{\theta}_{\mathrm{c}}\right)$ and $\hat{h}_{\mathrm{m}_{\mathrm{c}}}=\hat{h}_{\mathrm{m}_{\mathrm{c}}}(U)=h_{\mathrm{m}}\left(\hat{\theta}_{\mathrm{c}}\right)$, are 

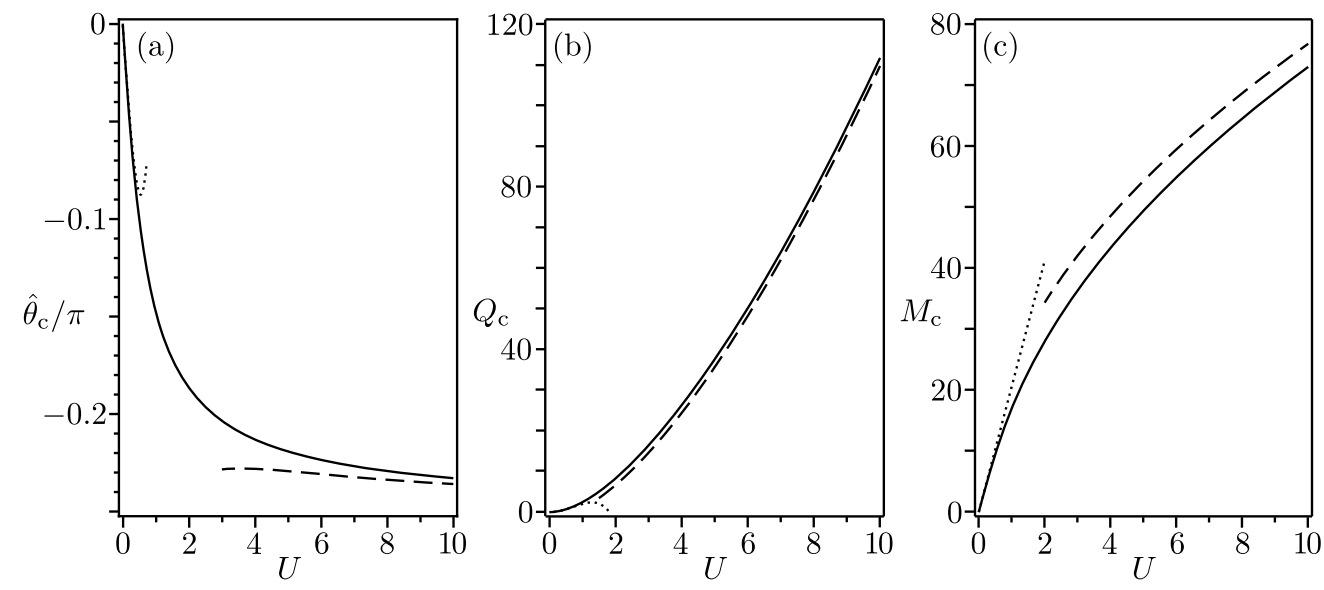

Figure 4. (a) The scaled position of the corner in the critical solution $\hat{\theta}_{\mathrm{c}} / \pi$, (b) the critical flux $Q_{\mathrm{c}}$, and (c) the critical load $M_{\mathrm{c}}$ plotted as functions of $U$ (solid lines) together with the asymptotic solutions (3.9), (3.10) and (3.15), and (3.16), (3.17) and (3.34) in the limits $U \rightarrow 0$ (dotted lines) and $U \rightarrow \infty$ (dashed lines), respectively. Full-ring solutions exist only for values of $Q, U$ and $M$ that lie below the solid curves in (b) and (c).

then given by

$$
\hat{a}_{\mathrm{c}}=\frac{\hat{B}_{\mathrm{c}}}{\hat{m}_{\mathrm{c}}}=\frac{\hat{B}_{\mathrm{c}}}{\left(-\sin \hat{\theta}_{\mathrm{c}}\right)^{1 / 2}}
$$

and

$$
\hat{h}_{\mathrm{m}_{\mathrm{c}}}=\frac{1}{\hat{m}_{\mathrm{c}}} \tan \frac{\hat{B}_{\mathrm{c}}}{2}=\frac{1}{\left(-\sin \hat{\theta}_{\mathrm{c}}\right)^{1 / 2}} \tan \frac{\hat{B}_{\mathrm{c}}}{2},
$$

respectively, and from (2.18) and (3.1) the critical flux $Q_{\mathrm{c}}=Q_{\mathrm{c}}(U)$ is given by

$$
Q_{\mathrm{c}}=\frac{F\left(\hat{B}_{\mathrm{c}}\right)}{9 \sin ^{2} \hat{\theta}_{\mathrm{c}} \cos \hat{\theta}_{\mathrm{c}}} .
$$

Note that in the special case $U=1$ we obtain the exact values $\hat{B}_{\mathrm{c}}=\pi / 2, \hat{\theta}_{\mathrm{c}}=-\cot ^{-1} 2 \simeq$ $-0.46365, \hat{a}_{\mathrm{c}}=5^{1 / 4} \pi / 2 \simeq 2.34889, \hat{h}_{\mathrm{m}_{\mathrm{c}}}=5^{1 / 4} \simeq 1.49535$ and $Q_{\mathrm{c}}=10 \sqrt{5} / 9 \simeq 2.48452$.

With the rotation speed $U$ prescribed and the value of $Q_{c}$ obtained from (3.7), the solution for the critical semi-width $a_{\mathrm{c}}$, critical ring profile $h_{\mathrm{c}}$, critical maximum thickness $h_{\mathrm{mc}}=h_{\mathrm{c}}(\theta, 0)$, critical pressure $p_{\mathrm{c}}$, critical velocity $u_{\mathrm{c}}$ and critical load $M_{\mathrm{c}}$ may be obtained from (2.18), (2.10), (2.11), (2.8), (2.15) and (2.28), respectively. Figure 4 shows (a) the scaled position of the corner in the critical solution $\hat{\theta}_{\mathrm{c}} / \pi$, (b) the critical flux $Q_{\mathrm{c}}$, and (c) the critical load $M_{\mathrm{c}}$, plotted as functions of $U$. In particular, figure 4 shows that $\hat{\theta}_{\mathrm{c}}$ is a decreasing function of $U$, decreasing from zero at $U=0$ to $-\pi / 4$ as $U \rightarrow \infty$. Figure 4 also shows that both the critical flux $Q_{\mathrm{c}}$ and critical load $M_{\mathrm{c}}$ are increasing functions of $U$, increasing from zero at $U=0$ to infinity as $U \rightarrow \infty$, and so full-ring solutions, which exist only for values of $Q, U$ and $M$ that lie below the solid curves in figures 4(b) and 4(c), exist for a wider range of fluxes and loads when the rotation speed is increased.

Figure 5 shows (a) the critical semi-width $a_{\mathrm{c}}$ and (b) the critical maximum thickness $h_{\mathrm{mc}}$ plotted as functions of $\theta / \pi$ for a range of values of $U$, and figure 6 shows the same quantities plotted as functions of $U$ for a range of values of $\theta$. In particular, figures 5 and 6 show that both $a_{\mathrm{c}}$ and $h_{\mathrm{mc}}$ are increasing functions of $U$ (as is the critical ring profile $h_{\mathrm{c}}$ ). 

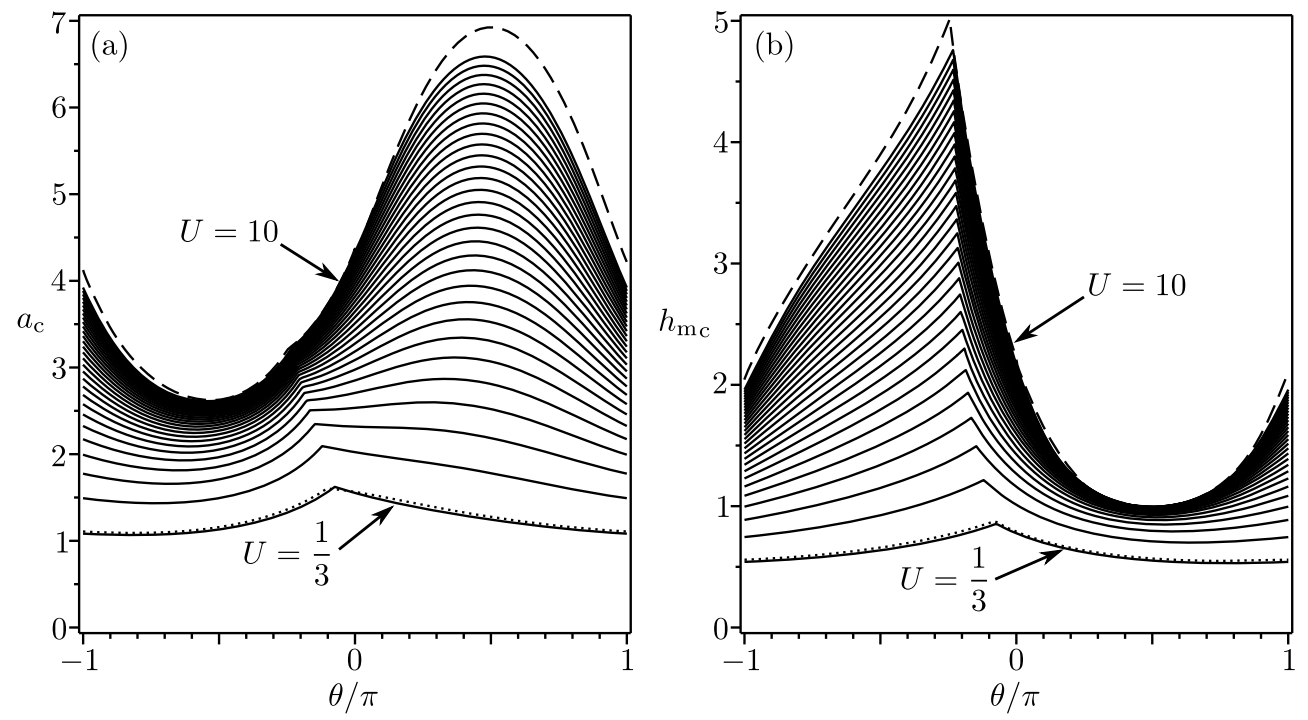

Figure 5. (a) The critical semi-width $a_{\mathrm{c}}$ and (b) the critical maximum thickness $h_{\mathrm{mc}}$ plotted as functions of $\theta / \pi$ for $U=1 / 3,2 / 3,1, \ldots, 10$ (solid lines) together with the asymptotic solutions (3.11) and (3.12), and (3.24), (3.25), (3.30) and (3.31) in the limits $U \rightarrow 0$ (dotted lines) and $U \rightarrow \infty$ (dashed lines) for $U=1 / 3$ and $U=10$, respectively.
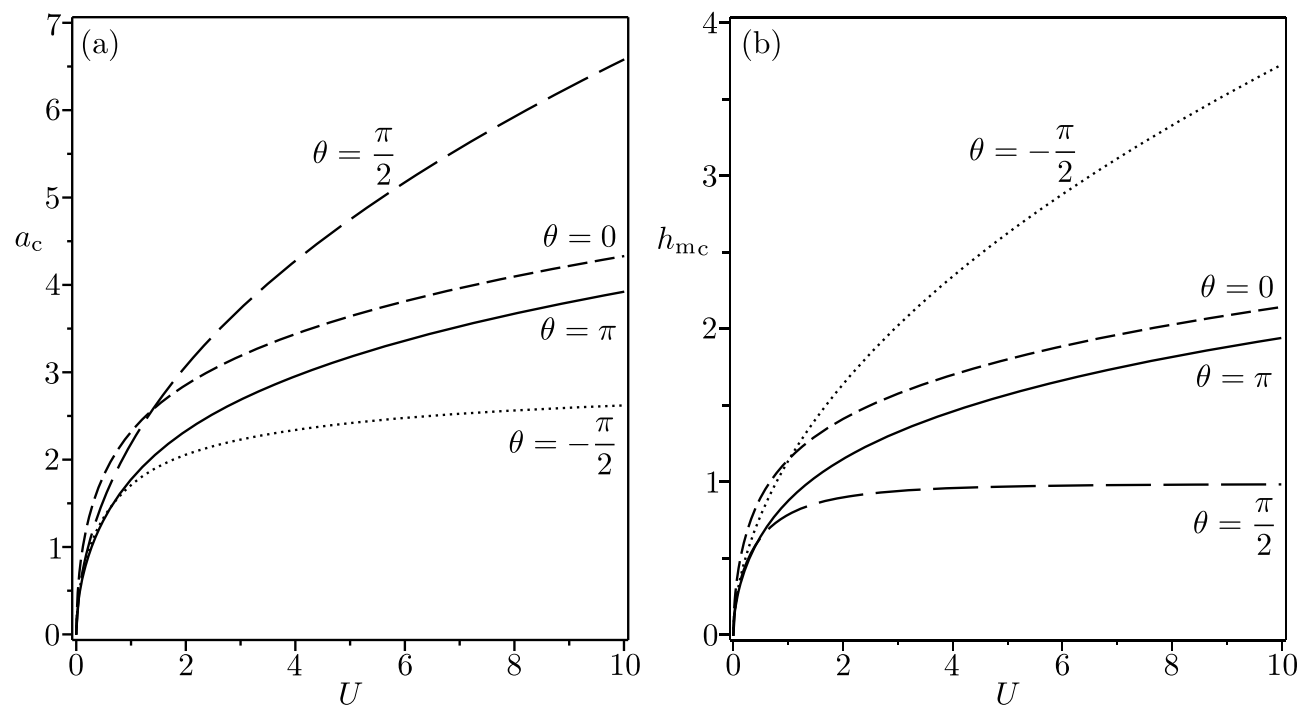

FiguRE 6. (a) The critical semi-width $a_{\mathrm{c}}$ and (b) the critical maximum thickness $h_{\mathrm{mc}}$ plotted as functions of $U$ for $\theta=\pi$ (solid lines), $\theta=\pi / 2$ (long-dashed lines), $\theta=0$ (short-dashed lines) and $\theta=-\pi / 2$ (dotted lines).

Figure 7 shows three-dimensional plots of the critical ring profile $h_{\mathrm{c}}$ for various values of $U$, clearly illustrating how the shape of the ring varies with $U$. In particular, figures $5-7$ show that when $U$ is small the ring is narrow and thin but not uniform, whereas when $U$ is large the ring is wide on the upper half of the cylinder and thick on the bottom half of the cylinder. In Subsections 3.1 and 3.2 below we will analyse the behaviour of the critical solution in the asymptotic limits $U \rightarrow 0$ and $U \rightarrow \infty$, respectively. 


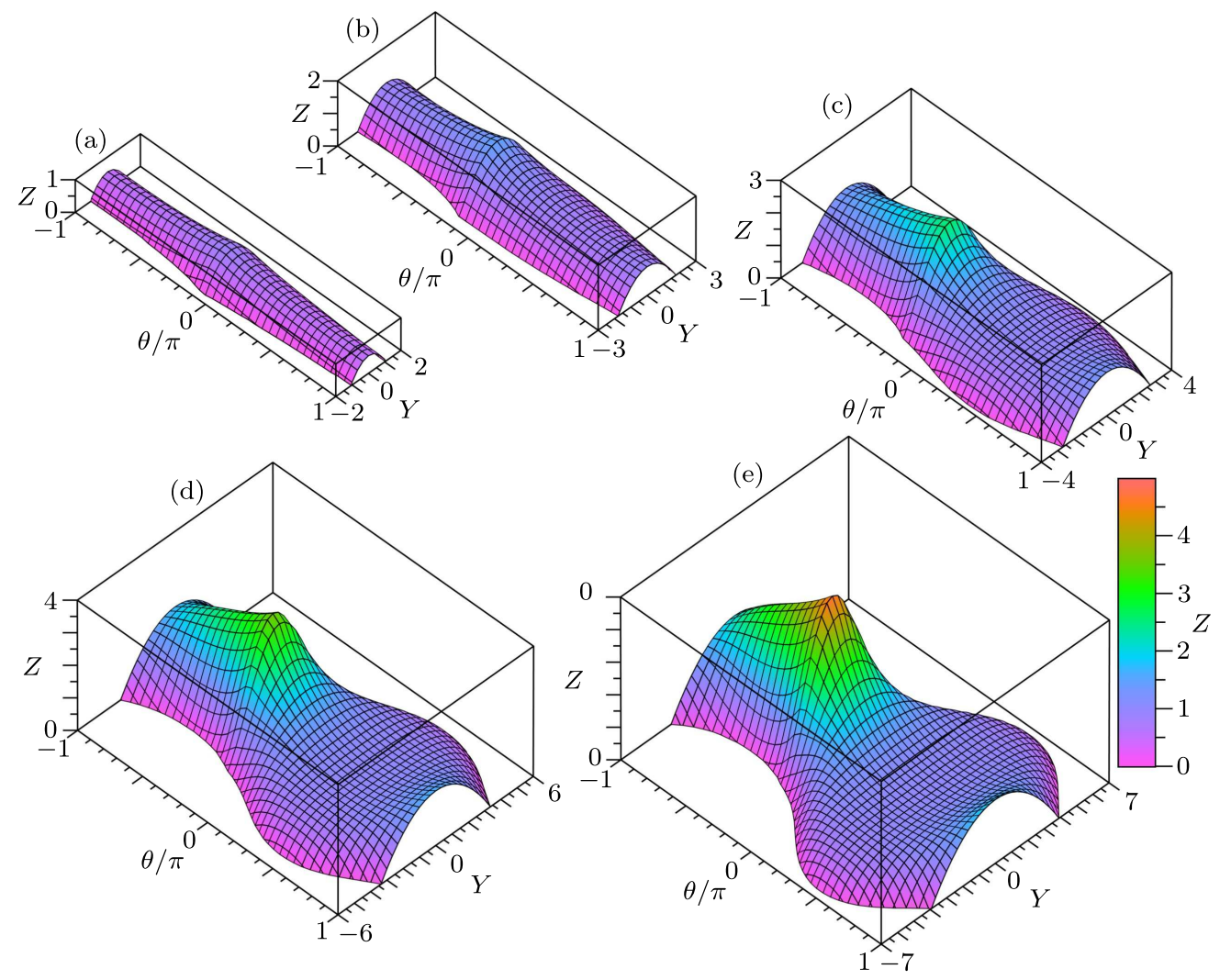

Figure 7. Three-dimensional plots of the critical ring profile $h_{\mathrm{c}}$ as a function of $Y=a_{\mathrm{c}} y$ and $\theta / \pi$ for (a) $U=1 / 3$, (b) $U=1$, (c) $U=3$, (d) $U=6$ and (e) $U=10$.

Figures 3(b), 5 and 7 clearly illustrate that not only the position but also the shape of the corner in the critical solution vary with $U$. The slopes of the critical semi-width $a_{\mathrm{c}}$, the rivulet profile $h_{\mathrm{c}}$, and the critical maximum thickness $h_{\mathrm{mc}}$ on either side of the corner (i.e. as $\theta \rightarrow \hat{\theta}_{\mathrm{c}}^{ \pm}$) are denoted by

$$
A_{\mathrm{c}}^{ \pm}=\left.\frac{\mathrm{d} a_{\mathrm{c}}}{\mathrm{d} \theta}\right|_{\theta=\hat{\theta}_{\mathrm{c}}^{ \pm}}, \quad H_{\mathrm{c}}^{ \pm}=\left.\frac{\partial h_{\mathrm{c}}}{\partial \theta}\right|_{\theta=\hat{\theta}_{\mathrm{c}}^{ \pm}} \quad \text { and } \quad H_{\mathrm{mc}}^{ \pm}=\left.H_{\mathrm{c}}^{ \pm}\right|_{y=0}=\left.\frac{\mathrm{d} h_{\mathrm{mc}}}{\mathrm{d} \theta}\right|_{\theta=\hat{\theta}_{\mathrm{c}}^{ \pm}},
$$

respectively. Figure 8 shows (a) $A_{\mathrm{c}}^{ \pm}$and (b) $H_{\mathrm{m}_{\mathrm{c}}}^{ \pm}$plotted as functions of $U$. In particular, figure 8 shows that $A_{\mathrm{c}}^{-}$and $H_{\mathrm{m}_{\mathrm{c}}}^{-}$are positive increasing functions of $U$ (as is $H_{\mathrm{c}}^{-}$), whereas $H_{\mathrm{mc}}^{+}$is a negative decreasing function of $U$ (as is $H_{\mathrm{c}}^{+}$) and $A_{\mathrm{c}}^{+}$is a negative decreasing function of $U$ when $U<0.25452$, a negative increasing function of $U$ when $0.25452<U<1.40264$, and a positive increasing function of $U$ when $U>1.40264$. This behaviour of the corner in $h_{\mathrm{c}}$ differs significantly from that in the two-dimensional critical full-film solution described by Moffatt (1977), not only because the slopes vary with $U$ but also because the corner is not, in general, symmetric, as it is in the two-dimensional case. Of course, in practice the corner in the critical solution will not be perfectly sharp and higher-order effects will become significant very close to the corner (see Wilson et al. $(2002 b))$. 

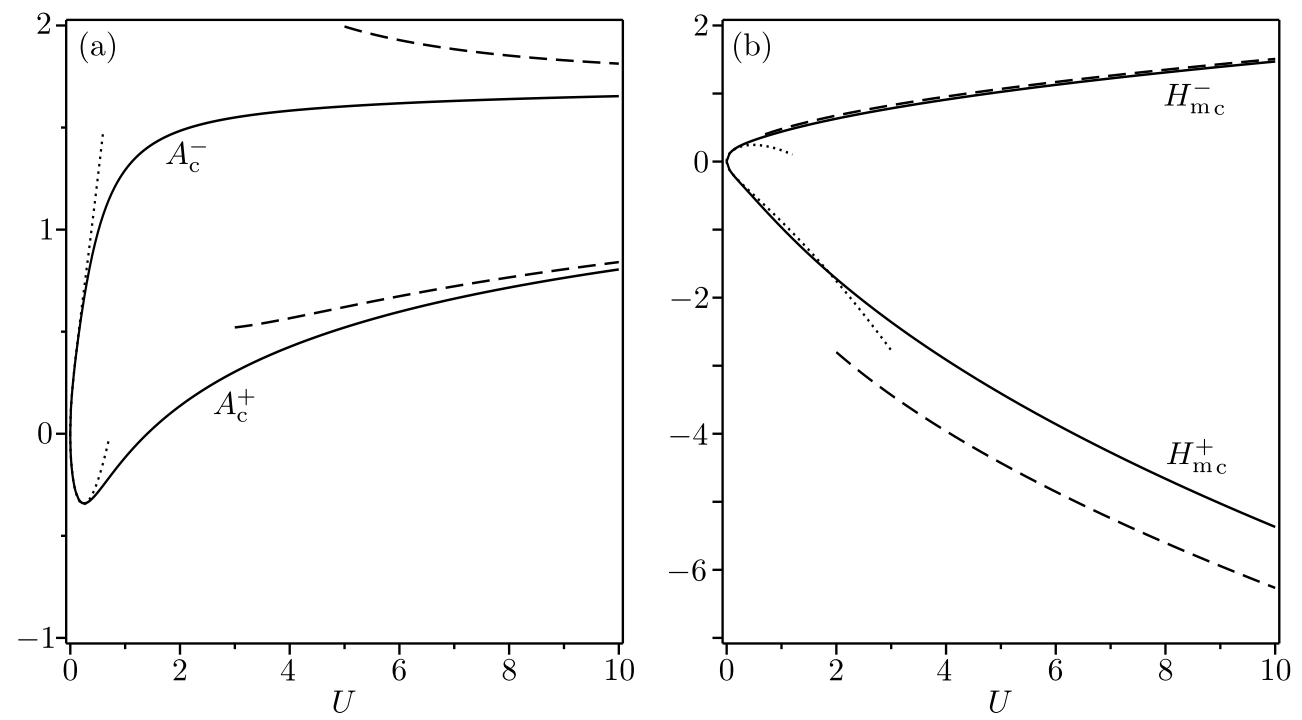

Figure 8. (a) The slopes of the critical semi-width $a_{\mathrm{c}}$ as $\theta \rightarrow \hat{\theta}_{\mathrm{c}}^{ \pm}, A_{\mathrm{c}}^{ \pm}$, and (b) the slopes of the critical maximum thickness $h_{\mathrm{m}_{\mathrm{c}}}$ as $\theta \rightarrow \hat{\theta}_{\mathrm{c}}^{ \pm}, H_{\mathrm{m}_{\mathrm{c}}}^{ \pm}$, plotted as functions of $U$ (solid lines) together with the asymptotic solutions (3.13) and (3.14), and (3.32) and (3.33) in the limits $U \rightarrow 0$ (dotted lines) and $U \rightarrow \infty$ (dashed lines), respectively.

\subsection{The limit of small rotation speed, $U \rightarrow 0$}

In the limit of small rotation speed, $U \rightarrow 0$, the position of the corner in the critical solution $\hat{\theta}_{\mathrm{c}}$ is given from (3.3) and (3.4) by

$$
\hat{\theta}_{\mathrm{c}}=-\frac{7 U}{9}+\frac{22148 U^{3}}{24057}+O\left(U^{5}\right),
$$

while from (3.7) the critical flux $Q_{\mathrm{c}}$ is given by

$$
Q_{\mathrm{c}}=\frac{35 U^{2}}{12}-\frac{1715 U^{4}}{1944}+O\left(U^{6}\right)
$$

These asymptotic solutions for $\hat{\theta}_{\mathrm{c}}$ and $Q_{\mathrm{c}}$ are shown as dotted lines in figures 4 (a) and $4(\mathrm{~b})$, respectively, and show that the effect of small rotation speed is to decrease $\hat{\theta}_{\mathrm{c}}$ and to increase $Q_{\mathrm{c}}$ from zero at $U=0$.

With the solution for $Q_{\mathrm{c}}$ known it may be shown from (2.18), (2.21) and (2.22) that the critical semi-width $a_{\mathrm{c}}$ is given by

$$
\begin{aligned}
& a_{\mathrm{c}}=\frac{1}{2}\left(\frac{35 U}{1+\sqrt{2} S \sin (\theta / 2)}\right)^{1 / 2} \\
& \quad+\frac{7 \sqrt{35} S \cos (\theta / 2)(5 S \sin (\theta / 2)-\sqrt{2})}{72(1+\sqrt{2} S \sin (\theta / 2))^{3 / 2}} U^{3 / 2}+O\left(U^{5 / 2}\right),
\end{aligned}
$$



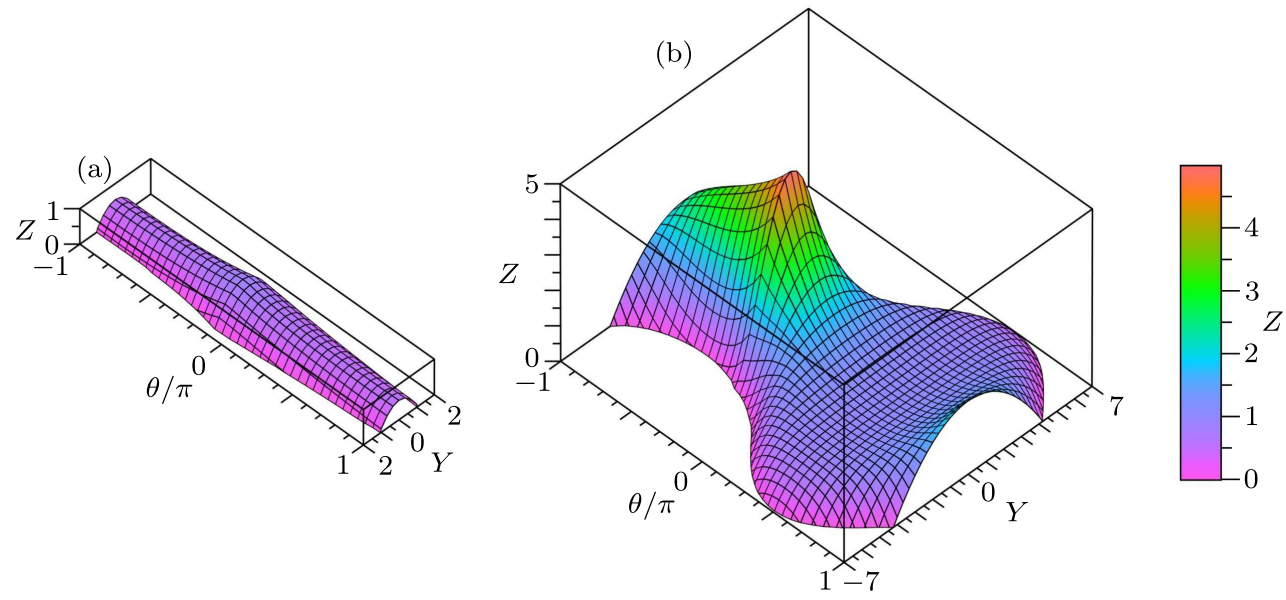

Figure 9. Three-dimensional plots of the asymptotic solutions for the critical ring profile $h_{\mathrm{c}}$ in the limit (a) $U \rightarrow 0$, given by (3.12), for $U=1 / 3$ and (b) $U \rightarrow \infty$, given by (3.30) and (3.31), for $U=10$, and plotted as functions of $Y=a_{\mathrm{c}} y$ and $\theta / \pi$. These plots may be compared with the corresponding exact solutions shown in figures $7(\mathrm{a})$ and $7(\mathrm{e})$, respectively.

and hence from (2.10) and (2.12) that the critical ring profile $h_{\mathrm{c}}$ is given by

$$
\begin{aligned}
h_{\mathrm{c}}= & \frac{1-y^{2}}{4}\left(\frac{35 U}{1+\sqrt{2} S \sin (\theta / 2)}\right)^{1 / 2} \\
& +\frac{7 \sqrt{35} S\left(1-y^{2}\right) \cos (\theta / 2)\left(5 S \sin (\theta / 2)\left(3 y^{2}-1\right)-2 \sqrt{2}\right)}{288(1+\sqrt{2} S \sin (\theta / 2))^{3 / 2}} U^{3 / 2}+O\left(U^{5 / 2}\right),
\end{aligned}
$$

where we have defined $S=\operatorname{sgn}\left(\theta-\hat{\theta}_{\mathrm{c}}\right)$. Figure $9($ a) shows a three-dimensional plot of the asymptotic solution for the critical ring profile $h_{\mathrm{c}}$ given by (3.12) for $U=1 / 3$, which may be compared with the corresponding exact solution shown in figure $7(\mathrm{a})$. The asymptotic solutions for the critical semi-width $a_{\mathrm{c}}$ and the critical maximum thickness $h_{\mathrm{mc}}$ are shown as dotted lines in figure 5. In particular, these solutions show that the effect of small rotation speed is to increase both $a_{\mathrm{c}}$ and $h_{\mathrm{c}}$ from zero at $U=0$.

The slopes on either side of the corners in $a_{\mathrm{c}}$ and $h_{\mathrm{c}}, A_{\mathrm{c}}^{ \pm}$and $H_{\mathrm{c}}^{ \pm}$, are given by

$$
A_{\mathrm{c}}^{ \pm}=\mp \frac{(70 U)^{1 / 2}}{8}+\frac{(35 U)^{3 / 2}}{144}+O\left(U^{5 / 2}\right)
$$

and

$$
H_{\mathrm{c}}^{ \pm}=\mp \frac{(70 U)^{1 / 2}\left(1-y^{2}\right)}{16}+\frac{(35 U)^{3 / 2}\left(1-y^{2}\right)\left(3 y^{2}-1\right)}{576}+O\left(U^{5 / 2}\right) .
$$

The asymptotic solutions for $A_{\mathrm{c}}^{ \pm}$and $H_{\mathrm{m}_{\mathrm{c}}}^{ \pm}$are shown as dotted lines in figure 8 , and show that the effect of small rotation speed is to increase $A_{\mathrm{c}}^{-}$and $H_{\mathrm{c}}^{-}$and to decrease $A_{\mathrm{c}}^{+}$and $H_{\mathrm{c}}^{+}$from zero at $U=0$.

The critical load $M_{\mathrm{c}}$ is given by

$$
M_{\mathrm{c}}=\frac{70 U}{3} \log (1+\sqrt{2})+O\left(U^{3}\right) .
$$

This asymptotic solution is shown as a dotted line in figure $4(\mathrm{c})$, and shows that the effect of small rotation speed is to increase $M_{\mathrm{c}}$ from zero at $U=0$. 
(a)
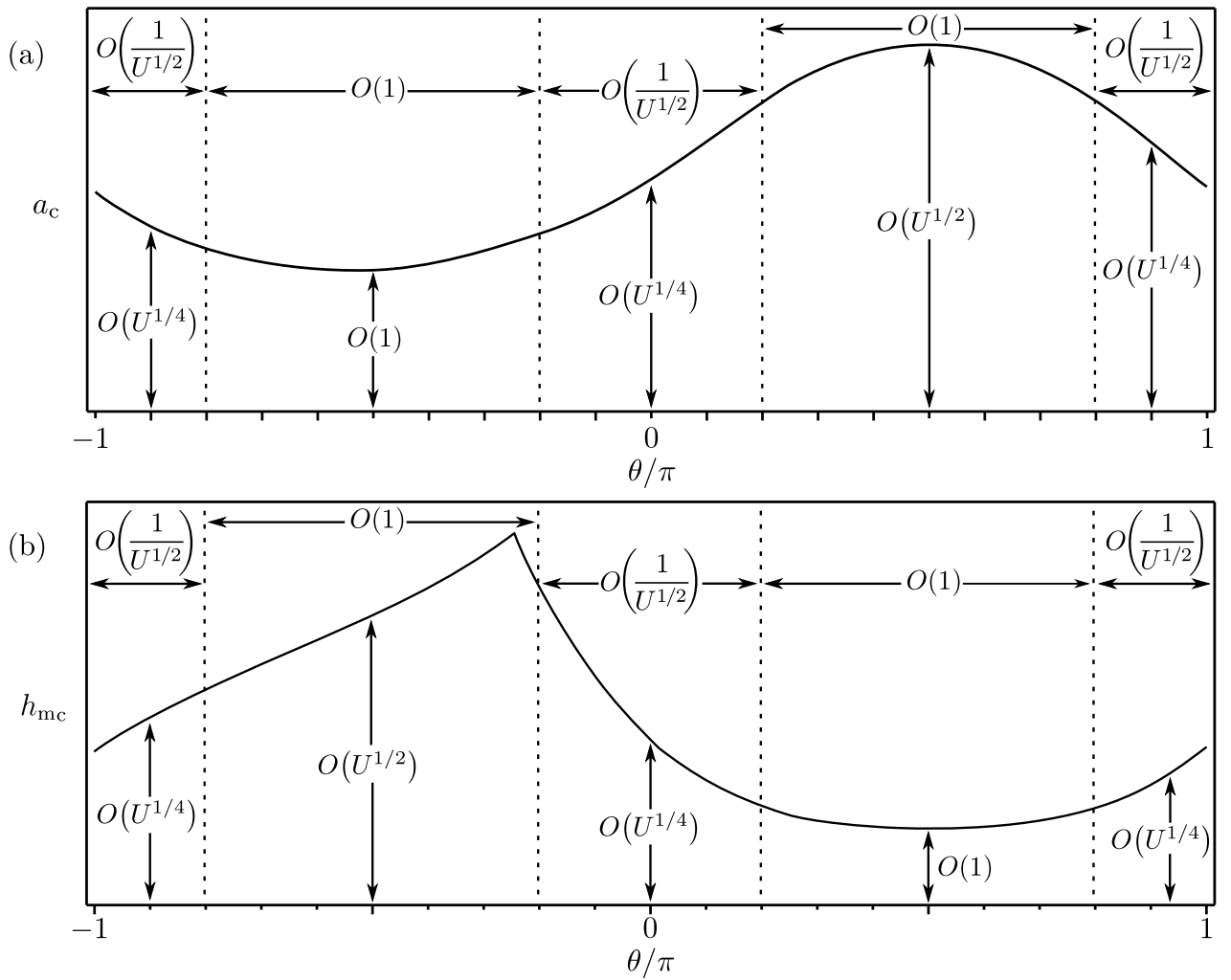

FIGURE 10. Sketches showing the structure of the asymptotic solutions for (a) the critical semi-width $a_{\mathrm{c}}$ and (b) the critical maximum thickness $h_{\mathrm{m}_{\mathrm{c}}}$ (the latter of which is also typical of $h_{\mathrm{c}}$ away from $y= \pm 1$ ) in the limit $U \rightarrow \infty$.

\subsection{The limit of large rotation speed, $U \rightarrow \infty$}

In the limit of large rotation speed, $U \rightarrow \infty$, the position of the corner in the critical solution $\hat{\theta}_{\mathrm{c}}$ is given from (3.3) and (3.4) by

$$
\hat{\theta}_{\mathrm{c}}=-\frac{\pi}{4}+\frac{3}{4 U}-\frac{15}{8 \pi}\left(\frac{5}{2 U^{3}}\right)^{1 / 2}+O\left(\frac{1}{U^{2}}\right)
$$

while from (3.7) the critical flux $Q_{\mathrm{c}}$ is given by

$$
Q_{\mathrm{c}}=\frac{8 \pi U^{3 / 2}}{3 \sqrt{5}}-\frac{2 \pi U^{1 / 2}}{\sqrt{5}}+O(1)
$$

These asymptotic solutions for $\hat{\theta}_{\mathrm{c}}$ and $Q_{\mathrm{c}}$ are shown as dashed lines in figures $4(\mathrm{a})$ and 4(b), respectively, and show that the effect of large but finite rotation speed is to increase $\hat{\theta}_{\mathrm{c}}$ from its $O(1)$ leading order value and to make $Q_{\mathrm{c}}$ large (specifically, $Q_{\mathrm{c}}=O\left(U^{3 / 2}\right)$ ).

Unlike in the limit $U \rightarrow 0$, the asymptotic solutions for the critical semi-width $a_{\mathrm{c}}$ and the critical ring profile $h_{\mathrm{c}}$ in the limit $U \rightarrow \infty$ are qualitatively different for positive and negative values of $\theta$ (i.e. on the upper and lower halves of the cylinder) and have thin boundary layers of width $O\left(1 / U^{1 / 2}\right) \ll 1$ near $\theta=0$ and $\theta=\pi$. Sketches showing the structure of the asymptotic solutions for $a_{\mathrm{c}}$ and $h_{\mathrm{m}_{\mathrm{c}}}$ (the latter of which is also typical of $h_{\mathrm{c}}$ away from $y= \pm 1$ ) in the limit $U \rightarrow \infty$ are shown in figure 10 . 


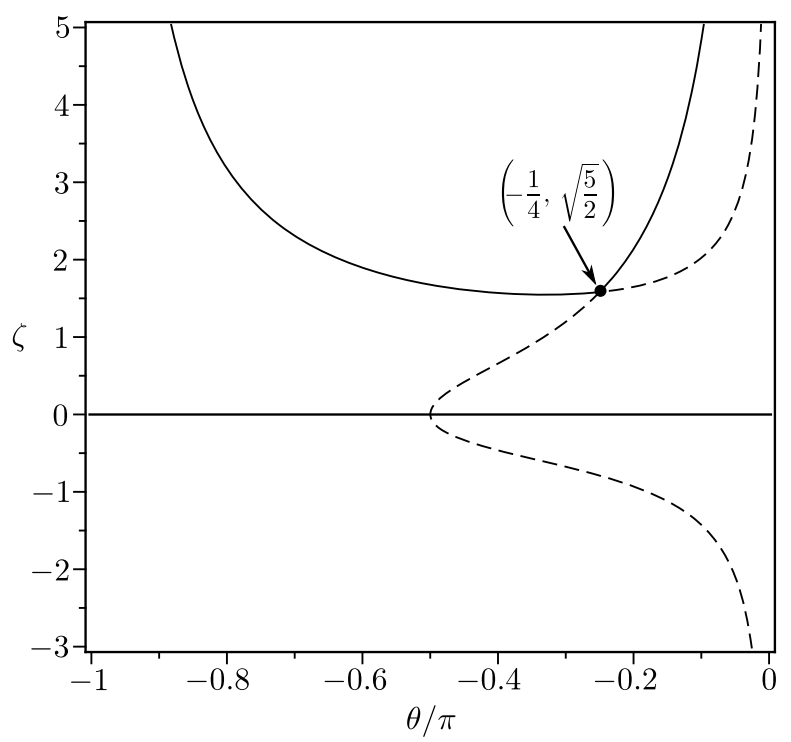

Figure 11. Plot of the real roots of the cubic equation (3.20) $\zeta$ as a function of $\theta / \pi$. The largest positive real root given by (3.21), which corresponds to the full-ring solution, is shown with a solid line, while the other real roots, which correspond to higher branches of solutions, are shown with dashed lines.

Away from the boundary layers near $\theta=0$ and $\theta=\pi$, the solution for the critical semi-width $a_{\mathrm{c}}$ is given by

$$
a_{\mathrm{c}}=\frac{4 \pi m U^{1 / 2}}{3 \sqrt{5}}+\frac{1}{m}+O\left(\frac{1}{U^{1 / 2}}\right)
$$

if $0<\theta<\pi$ and

$$
a_{\mathrm{c}}=\frac{\pi}{m}-\frac{\zeta}{m U^{1 / 2}}+O\left(\frac{1}{U^{3 / 2}}\right)
$$

if $-\pi<\theta<0$, where $\zeta=\zeta(\theta)$ is a root of the cubic polynomial equation

$$
8 \sin ^{2} \theta \zeta^{3}+6 \sqrt{5} \sin \theta \zeta^{2}+5 \sqrt{5} \cos \theta=0
$$

that is real in the interval $-\pi<\theta<0$. A plot of the real roots of (3.20) is shown in figure 11. The largest positive real root, which corresponds to the full-ring solution, is shown with a solid line, while the other real roots, which correspond to higher branches of solutions, are shown with dashed lines. The largest positive real root of (3.20) may be written explicitly as

$$
\zeta= \begin{cases}-\frac{\sqrt{5}}{4 \sin \theta}\left[1+2 \cosh \left(\frac{1}{3} \cosh ^{-1}[1+2 \sin 2 \theta]\right)\right] & \text { if }-\pi<\theta<-\frac{\pi}{2}, \\ \frac{3 \sqrt{5}}{4} & \text { if } \theta=-\frac{\pi}{2}, \\ -\frac{\sqrt{5}}{4 \sin \theta}\left[1+2 \cos \left(\frac{1}{3} \cos ^{-1}[1+2 \sin 2 \theta]\right)\right] & \text { if }-\frac{\pi}{2}<\theta<0,\end{cases}
$$

which (in agreement with the behaviour of $\hat{\theta}_{\mathrm{c}}$ in the limit $U \rightarrow \infty$ described previously) has a corner at $\theta=-\pi / 4$. From (3.18) and (3.19) we see that the effect of large but finite rotation speed is to make $a_{\mathrm{c}}$ large (specifically, $a_{\mathrm{c}}=O\left(U^{1 / 2}\right)$ ) on the upper half 


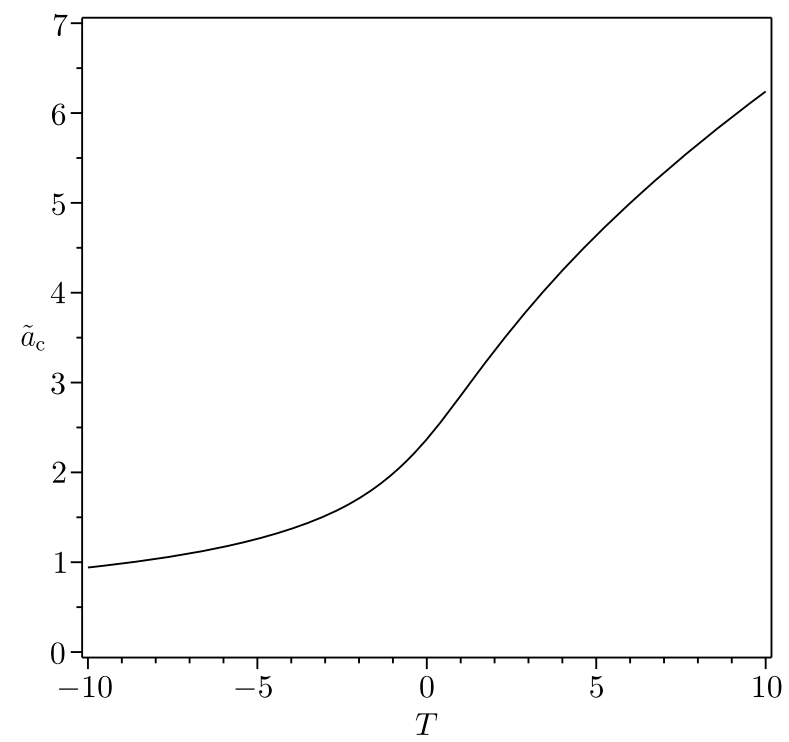

Figure 12. Plot of $\tilde{a}_{\mathrm{c}}$ as a function of $T$ obtained from (3.23).

of the cylinder and to decrease it from its $O(1)$ leading order value on the lower half of the cylinder. Note that there is no corner at leading order in the solution for $a_{\mathrm{c}}$ given by (3.18) and (3.19); the corner does, however, appear at first order. In the boundary layers near $\theta=0$ and $\theta=\pi, a_{\mathrm{c}}$ is of $O\left(U^{1 / 4}\right)$ (which is intermediate between $O(1)$ and $\left.O\left(U^{1 / 2}\right)\right)$ and is given by

$$
a_{\mathrm{c}}= \begin{cases}\tilde{a}_{\mathrm{c}} U^{1 / 4}-\operatorname{sgn}(\cos \theta) \frac{F\left(\tilde{a}_{\mathrm{c}}|T|^{1 / 2}\right)}{9 U^{1 / 4}|T|^{3 / 2} G^{\prime}\left(\tilde{a}_{\mathrm{c}}|T|^{1 / 2}\right)}+O\left(\frac{1}{U^{3 / 4}}\right) & \text { if } \theta \neq 0 \text { and } \theta \neq \pi, \\ \frac{2 \sqrt{\pi} U^{1 / 4}}{5^{1 / 4}}+\operatorname{sgn}(\cos \theta) \frac{8 \pi^{3 / 2}}{35(125 U)^{1 / 4}}+O\left(\frac{1}{U^{3 / 4}}\right) & \text { if } \theta=0 \text { or } \theta=\pi,\end{cases}
$$

where $\tilde{a}_{\mathrm{c}}=\tilde{a}_{\mathrm{c}}(T)$ is the smallest positive solution of

$$
G\left(\tilde{a}_{\mathrm{c}}|T|^{1 / 2}\right)=\frac{8 \pi|T|}{3 \sqrt{5}}
$$

and $T(\neq 0)$ is given by $T=\theta U^{1 / 2}$ with $\tilde{a}_{\mathrm{c}}=a_{\mathrm{c} 0}$ in the boundary layer near $\theta=0$ and by $T=(\operatorname{sgn}(\theta) \pi-\theta) U^{1 / 2}$ with $\tilde{a}_{\mathrm{c}}=a_{\mathrm{c} \pi}$ in the boundary layer near $\theta=\pi$. A plot of $\tilde{a}_{\mathrm{c}}$ as a function of $T$ obtained from (3.23) is shown in figure 12. A composite solution for $a_{\mathrm{c}}$ may be written as

$$
a_{\mathrm{c}}=\frac{4 \pi U^{1 / 2}}{3 \sqrt{5}}\left[m-(\pi-\theta)^{1 / 2}-\theta^{1 / 2}\right]+\frac{1}{m}-\frac{1}{(\pi-\theta)^{1 / 2}}-\frac{1}{\theta^{1 / 2}}+\left(a_{\mathrm{c} 0}+a_{\mathrm{c} \pi}\right) U^{1 / 4}
$$

if $0<\theta<\pi$ and

$$
\begin{aligned}
& a_{\mathrm{c}}=\frac{\pi}{m}-\frac{\pi}{|\theta|^{1 / 2}}-\frac{\pi}{(\pi+\theta)^{1 / 2}} \\
& -\frac{1}{U^{1 / 2}}\left(\frac{\zeta}{m}-\frac{3 \sqrt{5}}{4}\left[\frac{1}{|\theta|^{3 / 2}}+\frac{1}{(\pi+\theta)^{3 / 2}}-\frac{8}{27|\theta|^{1 / 2}}+\frac{8}{27(\pi+\theta)^{1 / 2}}\right]\right)+\left(a_{\mathrm{c} 0}+a_{\mathrm{c} \pi}\right) U^{1 / 4}
\end{aligned}
$$


if $-\pi<\theta<0$, and is shown as a dashed line in figure $5(\mathrm{a})$.

Away from the boundary layers near $\theta=0$ and $\theta=\pi$, the solution for the critical ring profile $h_{\mathrm{c}}$ is given by

$$
h_{\mathrm{c}}=\xi+O\left(\frac{1-|y|}{U^{1 / 2}} \exp \left[-\frac{4 \pi m^{2} U^{1 / 2}(1-|y|)}{3 \sqrt{5}}\right]\right)
$$

if $0<\theta<\pi$, where $\xi=\xi(m)$ is given by

$$
\xi=\frac{1}{m}\left[1-2 \exp \left(-\left[\frac{4 \pi m^{2} U^{1 / 2}}{3 \sqrt{5}}+1\right]\right) \cosh \left(\left[\frac{4 \pi m^{2} U^{1 / 2}}{3 \sqrt{5}}+1\right] y\right)\right],
$$

and

$$
h_{\mathrm{c}}=\frac{1+\cos \pi y}{m \zeta} U^{1 / 2}+\frac{y \sin \pi y}{m}+O\left(\frac{1}{U^{1 / 2}}\right)
$$

if $-\pi<\theta<0$, where $\zeta$ is again given by (3.21). From (3.26) and (3.28) we see that the effect of large but finite rotation speed is to decrease $h_{\mathrm{c}}$ from its $O(1)$ leading order value on the upper half of the cylinder and to make it large (specifically, $h_{\mathrm{c}}=O\left(U^{1 / 2}\right)$ except near $y= \pm 1$ ) on the lower half of the cylinder. We note that, unlike in the asymptotic solution for $a_{\mathrm{c}}$ given by (3.18) and (3.19), the corner appears at leading order in the solution for $h_{\mathrm{c}}$ given by (3.26) and (3.28). In the boundary layers near $\theta=0$ and $\theta=\pi$, $h_{\mathrm{c}}$ is of $O\left(U^{1 / 4}\right)$ (i.e. of the same size as $a_{\mathrm{c}}$ ) and is given by

$$
h_{\mathrm{c}}=\eta(T, y)= \begin{cases}\frac{\cosh \left(\tilde{a}_{\mathrm{c}} T^{1 / 2}\right)-\cosh \left(\tilde{a}_{\mathrm{c}} T^{1 / 2} y\right)}{T^{1 / 2} \sinh \left(\tilde{a}_{\mathrm{c}} T^{1 / 2}\right)} U^{1 / 4}+O\left(\frac{1}{U^{1 / 4}}\right) & \text { if } 0<\theta<\pi, \\ \frac{\tilde{a}_{\mathrm{c}}\left(1-y^{2}\right)}{2} U^{1 / 4}+O\left(\frac{1}{U^{1 / 4}}\right) & \text { if } \theta=0 \text { or } \theta=\pi, \\ \frac{\cos \left(\tilde{a}_{\mathrm{c}}|T|^{1 / 2} y\right)-\cos \left(\tilde{a}_{\mathrm{c}}|T|^{1 / 2}\right)}{|T|^{1 / 2} \sin \left(\tilde{a}_{\mathrm{c}}|T|^{1 / 2}\right)} U^{1 / 4}+O\left(\frac{1}{U^{1 / 4}}\right) & \text { if }-\pi<\theta<0 .\end{cases}
$$

A composite solution for $h_{\mathrm{c}}$ may be written as

$$
h_{\mathrm{c}}=\xi(m)-\xi\left(\theta^{1 / 2}\right)-\xi\left([\pi-\theta]^{1 / 2}\right)+\eta\left(\theta U^{1 / 2}, y\right)+\eta\left([\pi-\theta] U^{1 / 2}, y\right)
$$

if $0<\theta<\pi$ and

$$
\begin{aligned}
h_{\mathrm{c}}= & \left.\frac{1}{m \zeta}-\frac{4}{3 \sqrt{5}}\left(|\theta|^{1 / 2}+(\pi+\theta)^{1 / 2}\right)\right](1+\cos \pi y) U^{1 / 2} \\
& +\left[\frac{1}{m}-\frac{1}{|\theta|^{1 / 2}}-\frac{1}{(\pi+\theta)^{1 / 2}}\right] y \sin \pi y+\eta\left(\theta U^{1 / 2}, y\right)+\eta\left([\pi+\theta] U^{1 / 2}, y\right)
\end{aligned}
$$

if $-\pi<\theta<0$, where $\zeta, \xi$ and $\eta$ are again given by (3.21), (3.27) and (3.29), respectively, and the corresponding composite solution for $h_{\mathrm{m}_{\mathrm{c}}}$ is shown as a dashed line in figure $5(\mathrm{~b})$. Figure $9(\mathrm{~b})$ shows a three-dimensional plot of the asymptotic solution for the critical ring profile $h_{\mathrm{c}}$ given by (3.30) and (3.31) for $U=10$, which may be compared with the corresponding exact solution shown in figure $7(\mathrm{e})$. The asymptotic solutions for the critical semi-width $a_{\mathrm{c}}$ and the critical maximum thickness $h_{\mathrm{m}_{\mathrm{c}}}$ are shown as dotted lines in figure 5 .

The slopes on either side of the corner in the solutions for $a_{\mathrm{c}}$ and $h_{\mathrm{c}}, A_{\mathrm{c}}^{ \pm}$and $H_{\mathrm{c}}^{ \pm}$, are given by

$$
A_{\mathrm{c}}^{ \pm}=\frac{\pi}{2^{3 / 4}}-\frac{2^{3 / 4} \sqrt{5}}{12 U^{1 / 2}}(9 \pm 2 \sqrt{6})+\frac{15 \pi}{2^{15 / 4} U}+O\left(\frac{1}{U^{3 / 2}}\right)
$$


and

$$
H_{\mathrm{c}}^{ \pm}=\mp \frac{2^{3 / 4} \sqrt{5} U^{1 / 2}}{30}(2 \sqrt{6} \pm 3)(1+\cos \pi y)+\frac{y \sin \pi y}{2^{3 / 4}}+O\left(\frac{1}{U^{1 / 2}}\right) .
$$

The asymptotic solutions for $A_{\mathrm{c}}^{ \pm}$and $H_{\mathrm{m}_{\mathrm{c}}}^{ \pm}$are shown as dashed lines in figure 8, and show that the effect of a large but finite rotation speed is to decrease $A_{\mathrm{c}}^{ \pm}$from their $O(1)$ leading order values and to make $H_{\mathrm{c}}^{ \pm}$large (specifically, $H_{\mathrm{c}}^{ \pm}=O\left(U^{1 / 2}\right)$ away from $y= \pm 1)$. Note that the leading order values of $A_{\mathrm{c}}^{ \pm}$are the same while the leading order values of $H_{\mathrm{c}}^{ \pm}$are of the same magnitude but opposite sign, confirming that there is no corner in the leading order solution for $a_{\mathrm{c}}$ but that there is a corner in the leading order solution for $h_{\mathrm{c}}$.

The critical load $M_{\mathrm{c}}$ is given by

$$
M_{\mathrm{c}}=\left(\frac{8 \pi^{2}}{3 \sqrt{5}}+\int_{-\pi}^{0} \frac{2 \pi}{m^{2} \zeta} \mathrm{d} \theta\right) U^{1 / 2}+O(1) \simeq 24.25391 U^{1 / 2}+O(1) .
$$

This asymptotic solution is shown as a dashed line in figure 4(c), and shows that the effect of a large but finite rotation speed is to make $M_{\mathrm{c}}$ large (specifically, $M_{\mathrm{c}}=O\left(U^{1 / 2}\right)$ ).

\section{The non-critical full-ring solution}

In Section 3 we described the behaviour of the critical full-ring solution when either the rotation speed $U$ or the load $M$ are prescribed. In this Section we describe the behaviour when both $U$ and $M$ are prescribed, in which case case a non-critical full-ring solution exists only provided that $U$ and $M$ satisfy $U>U_{\text {c }}$ (i.e. super-critical rotation speed) and $M<M_{\mathrm{c}}$ (i.e. sub-critical load) so that $Q<Q_{\mathrm{c}}$, i.e. only for values of $Q, U$ and $M$ that lie below the solid curves in figures $4(\mathrm{~b})$ and $4(\mathrm{c})$.

Figure 13 shows (a) the semi-width $a$ and (b) the maximum thickness $h_{\mathrm{m}}$ plotted as functions of $\theta / \pi$ for five different values of $M$ and a range of values of $U$ in each case (including the corresponding critical rotation speed $U_{\mathrm{c}}$ ), and figure 14 shows the same quantities plotted as functions of $\theta / \pi$ for three different values of $U$ and a range of values of $M$ in each case (including the corresponding critical load $M_{\mathrm{c}}$ ). In particular, figures 13 and 14 show that both $a$ and $h_{\mathrm{m}}$ are increasing functions of $M$ (as is the ring profile $h$ ) but are, in general, non-monotonic functions of $U$. Another feature evident from figures 13 and 14 is that while varying $M$ can have a significant effect on the shape of the ring, varying $U$ typically has relatively little effect, with the most significant changes occurring for values of $U$ close to $U_{\mathrm{c}}$ and for values of $\theta$ close to $\hat{\theta}_{\mathrm{c}}$. Figure 15 shows three-dimensional plots of the non-critical ring profile $h$ for various values of $U$ and $M$, clearly illustrating how the shape of the ring varies with both $U$ and $M$. In particular, figures 13-15 show that when $U$ is large the ring approaches a limiting non-uniform shape, whereas when $M$ is small the ring is narrow and thin with a uniform profile. In Subsections 4.1 and 4.2 below we will analyse the behaviour of the non-critical solution with prescribed rotation speed $U$ or prescribed load $M$ in the asymptotic limits $M \rightarrow 0$ and $U \rightarrow \infty$, respectively. Note that since, as we have already seen, full-ring solutions exist only when $M \leqslant M_{\mathrm{c}}$ and $U \geqslant U_{\mathrm{c}}$, there are no full-ring solutions in the limits $M \rightarrow \infty$ with prescribed $U$ and $U \rightarrow 0$ with prescribed $M$, and hence there is no corresponding analysis of the behaviour in these asymptotic limits. 

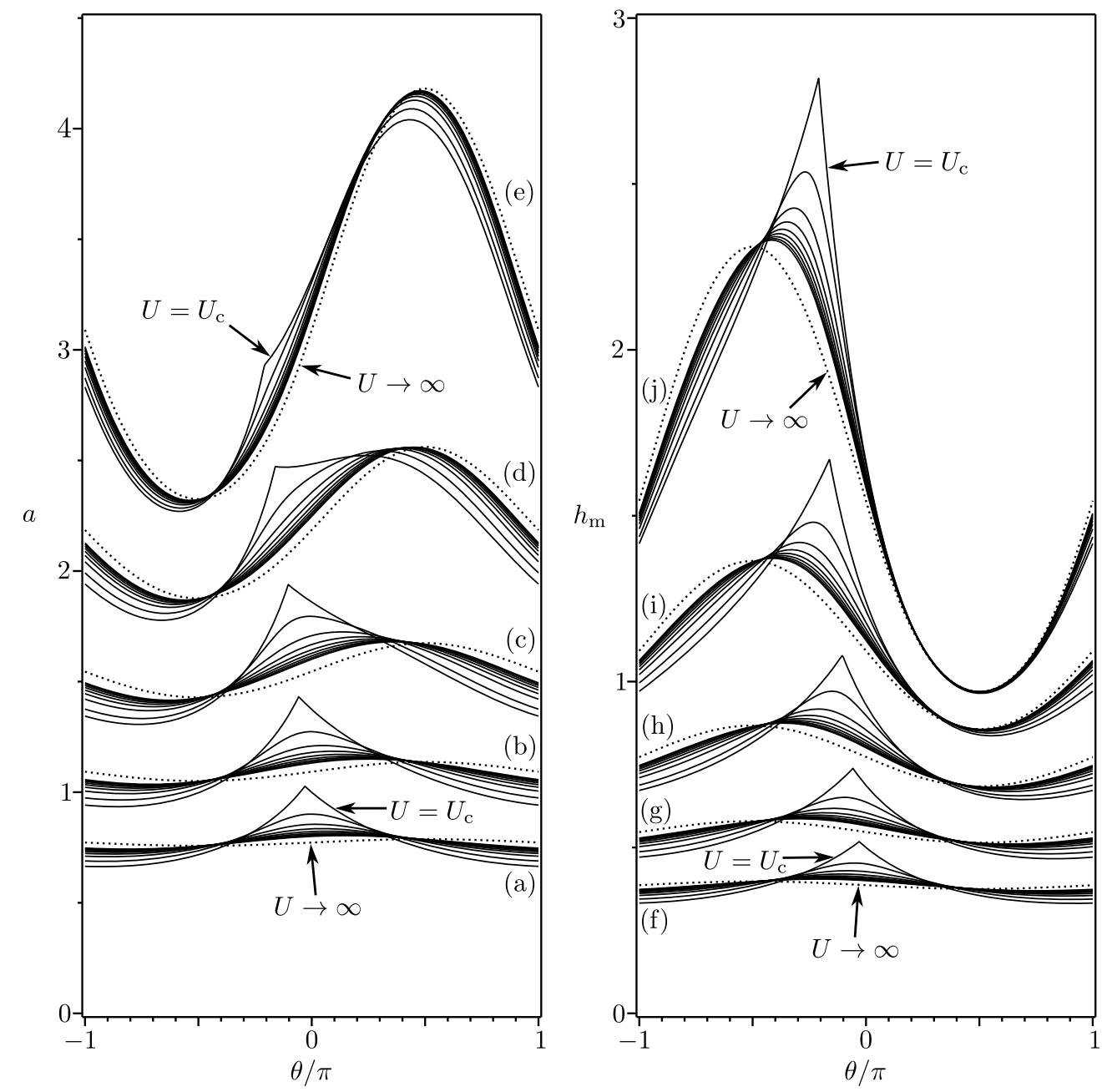

Figure 13. (a-e) The semi-width $a$ and $(\mathrm{f}-\mathrm{j})$ the maximum thickness $h_{\mathrm{m}}$ plotted as functions of $\theta / \pi$ for $(\mathrm{a}, \mathrm{f}) U=U_{\mathrm{c}} \simeq 0.12240$ and $U=0.15,0.2,0.25, \ldots, 0.5$ with $M=2.5,(\mathrm{~b}, \mathrm{~g})$ $U=U_{\mathrm{c}} \simeq 0.24834$ and $U=0.3,0.4,0.5, \ldots, 1$ with $M=5,(\mathrm{c}, \mathrm{h}) U=U_{\mathrm{c}} \simeq 0.52666$ and $U=0.6,0.8,1, \ldots, 2$ with $M=10,(\mathrm{~d}, \mathrm{i}) U=U_{\mathrm{c}} \simeq 1.24416$ and $U=1.5,2,2.5, \ldots, 5$ with $M=20$, and $(\mathrm{e}, \mathrm{j}) U=U_{\mathrm{c}} \simeq 3.51518$ and $U=4,5,6, \ldots, 11$ with $M=40$ (solid lines). The $O(1)$ leading order asymptotic solutions for $a$ and $h_{\mathrm{m}}$ in the limit $U \rightarrow \infty$ given by (4.2) and by (4.5)-(4.7), respectively, are shown as dotted lines.

\subsection{The limit of large rotation speed, $U \rightarrow \infty$}

In the limit of large rotation speed, $U \rightarrow \infty$, with the load $M$ prescribed, the flux $Q$ and the semi-width $a$ are given from (2.18) and (2.28) by

$$
Q=\frac{M U}{2 \pi}-\frac{I}{162 \pi U}+O\left(\frac{1}{U^{3}}\right)
$$

and

$$
a=a_{0}+\frac{\cos \theta F\left(m a_{0}\right)}{9 m^{3} G^{\prime}\left(m a_{0}\right) U}+O\left(\frac{1}{U^{2}}\right)
$$



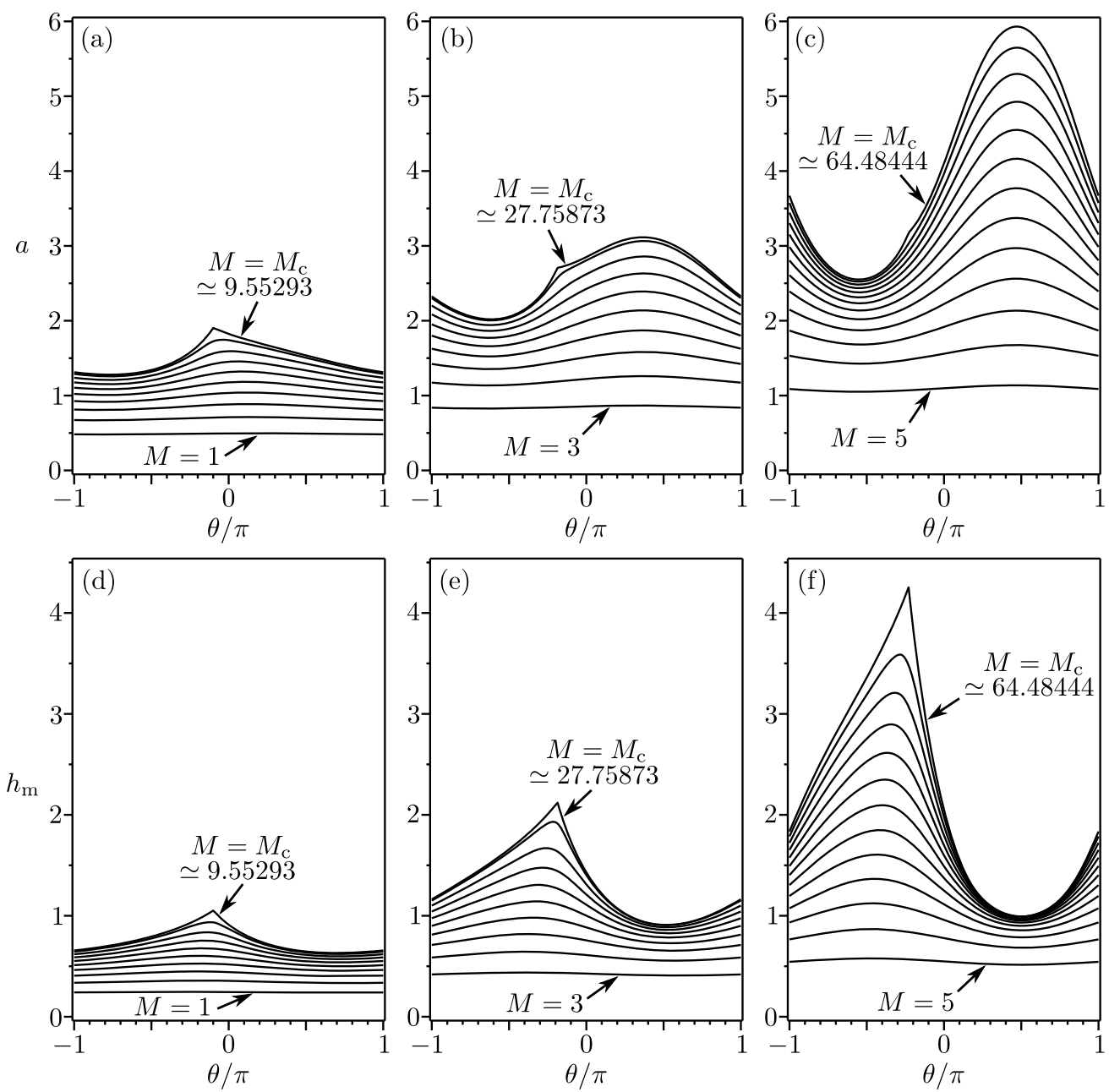

Figure 14. (a,b,c) The semi-width $a$ and $(\mathrm{d}, \mathrm{e}, \mathrm{f})$ the maximum thickness $h_{\mathrm{m}}$ plotted as functions of $\theta / \pi$ for $(\mathrm{a}, \mathrm{d}) M=1,2,3, \ldots, 9$ and $M=M_{\mathrm{c}} \simeq 9.55293$ with $U=1 / 2,(\mathrm{~b}, \mathrm{e}) M=3,6,9, \ldots$, 27 and $M=M_{\mathrm{c}} \simeq 27.75873$ with $U=2$ and (c,f) $M=5,10,15, \ldots, 60$ and $M=M_{\mathrm{c}} \simeq 64.48444$ with $U=8$.

where $a_{0}=a_{0}(\theta)$ is $a_{0}=(3 M / 4 \pi)^{1 / 2}$ when $\theta=0$ or $\theta=\pi$ and the smallest positive solution of

$$
G\left(m a_{0}\right)=\frac{m^{2} M}{2 \pi}
$$

when $\theta \neq 0$ and $\theta \neq \pi$, and $I=I(M)(>0)$, defined by

$$
I=\int_{-\pi}^{\pi} \frac{\cos ^{2} \theta F\left(m a_{0}\right) F^{\prime}\left(m a_{0}\right)}{m^{6} G^{\prime}\left(m a_{0}\right)} \mathrm{d} \theta
$$

is a monotonically increasing function of $M$ plotted in figure 16. Hence the ring profile 

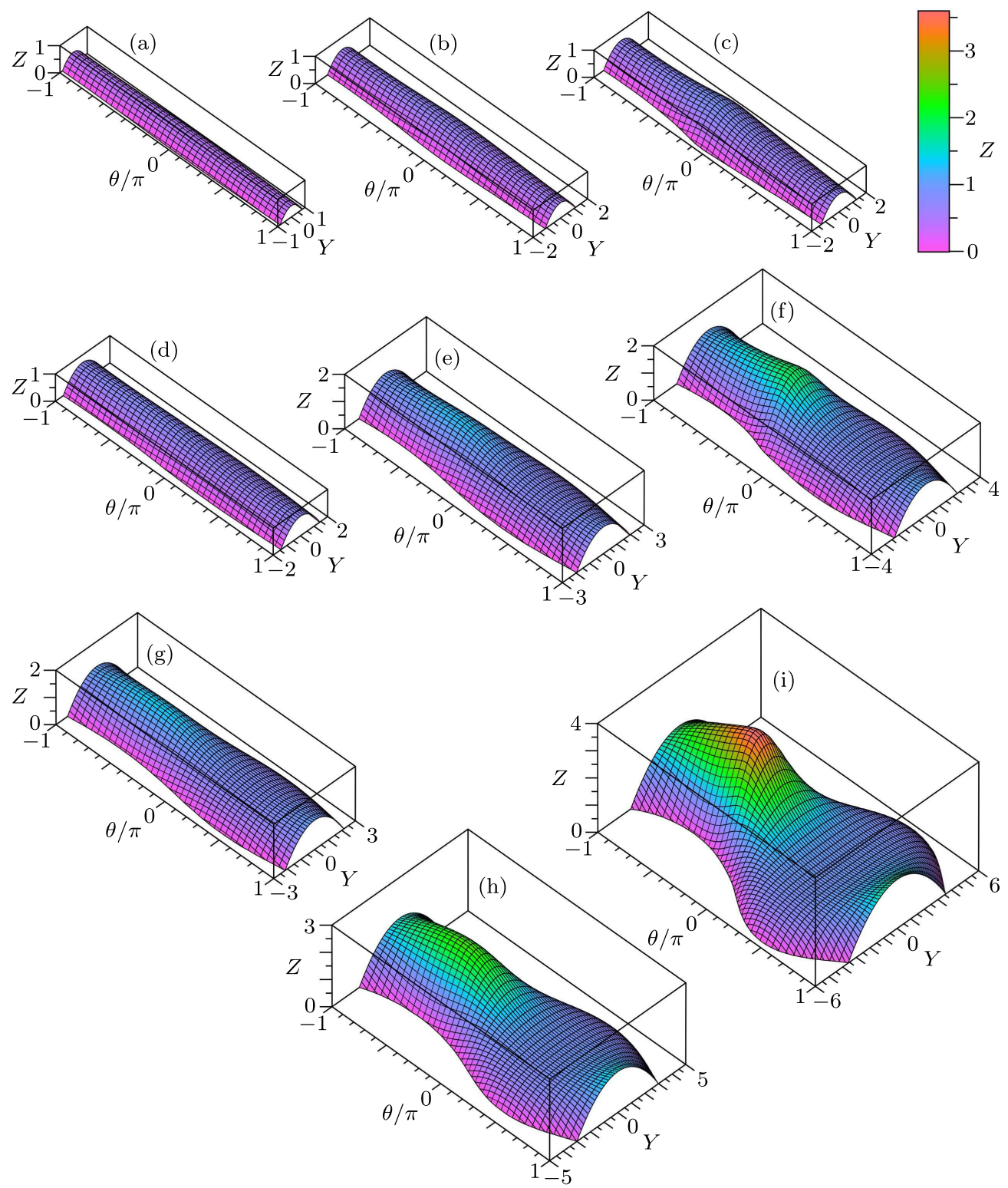

FiguRE 15. Three-dimensional plots of the non-critical ring profile $h$ as a function of $Y=a y$ and $\theta / \pi$ for (a) $M=3$, (b) $M=6$ and (c) $M=9$ for $U=1 / 2$, (d) $M=9$, (e) $M=18$ and (f) $M=27$ for $U=2$, and (g) $M=20$, (h) $M=40$ and (i) $M=60$ for $U=8$.

$h$ is given from (2.10) by

$$
\begin{aligned}
h & =\frac{\cosh m a_{0}-\cosh m a_{0} y}{m \sinh m a_{0}} \\
& +\frac{\cos \theta\left[\cosh m a_{0} \cosh m a_{0} y-y \sinh m a_{0} \sinh m a_{0} y-1\right] F\left(m a_{0}\right)}{9 m^{3} \sinh ^{2} m a_{0} G^{\prime}\left(m a_{0}\right) U}+O\left(\frac{1}{U^{2}}\right)
\end{aligned}
$$




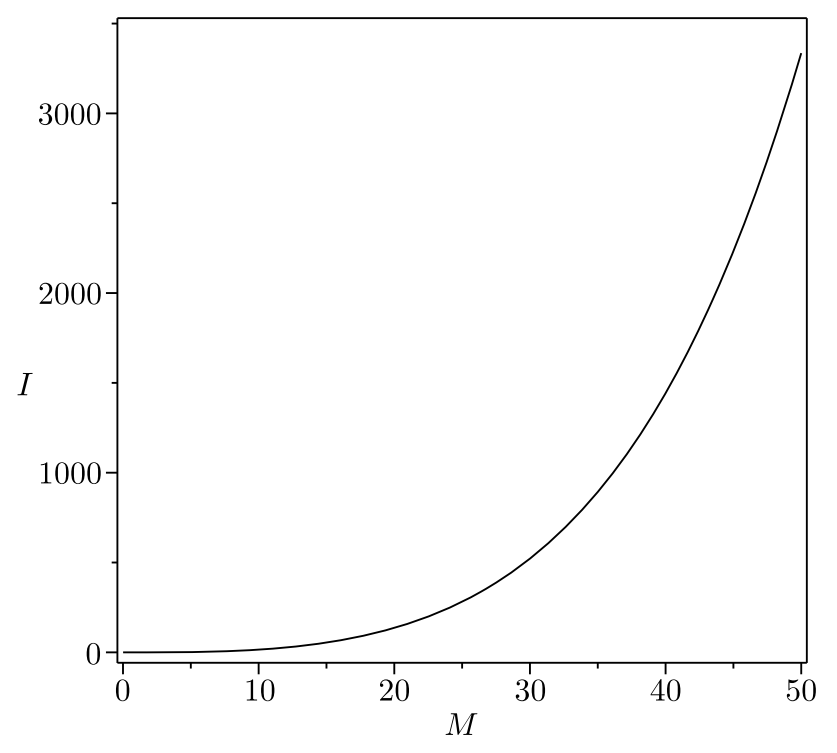

Figure 16. Plot of $I$ given by (4.4) as a function of $M$.

if $0<\theta<\pi$, by

$$
h=\frac{1-y^{2}}{2}\left(\frac{3 M}{4 \pi}\right)^{1 / 2}\left[1+\operatorname{sgn}(\cos \theta) \frac{3 M}{140 \pi U}\right]+O\left(\frac{1}{U^{2}}\right)
$$

if $\theta=0$ or $\theta=\pi$, and by

$$
\begin{aligned}
h= & \frac{\cos m a_{0} y-\cos m a_{0}}{m \sin m a_{0}} \\
& \quad-\frac{\cos \theta\left[\cos m a_{0} \cos m a_{0} y+y \sin m a_{0} \sin m a_{0} y-1\right] F\left(m a_{0}\right)}{9 m^{3} \sin ^{2} m a_{0} G^{\prime}\left(m a_{0}\right) U}+O\left(\frac{1}{U^{2}}\right)
\end{aligned}
$$

if $-\pi<\theta<0$. The asymptotic solution for the flux $Q$ given by (4.1) shows that the effect of large but finite rotation speed is to make $Q$ large (specifically, $Q=O(U)$ ). Figure 17 shows $Q$ plotted as a function of (a) $U$ for a range of values of $M$ and (b) $M$ for a range of values of $U$, respectively, together with the critical flux shown as a dashed line. Figure 17(a) also includes the asymptotic solution for $Q$ given by (4.1) shown as dotted lines and, rather unexpectedly, shows that in practice the asymptotic solution provides a surprisingly good approximation to $Q$ for all $U \geqslant U_{\text {c }}$. The $O(1)$ leading order asymptotic solutions for $a$ and $h_{\mathrm{m}}$ are shown as dotted lines in figure 13 for a range of values of $M$. Figure 18 shows three-dimensional plots of the asymptotic solution for the non-critical ring profile $h$ for various values of $U$ and $M$, which may be compared with the corresponding exact solutions shown in figures 15(c), 15(f) and 15(i). In particular, figure 18 shows that, like the asymptotic solution for $Q$, the asymptotic solution for $h$ provides a good approximation to the exact solution for all $U \geqslant U_{\mathrm{c}}$. In particular, the asymptotic solutions for $a$ and $h$ show that the effect of large but finite rotation speed is to increase (decrease) both $a$ and $h$ on the upward-moving (the downward-moving) side of the cylinder from their $O(1)$ leading order values. 

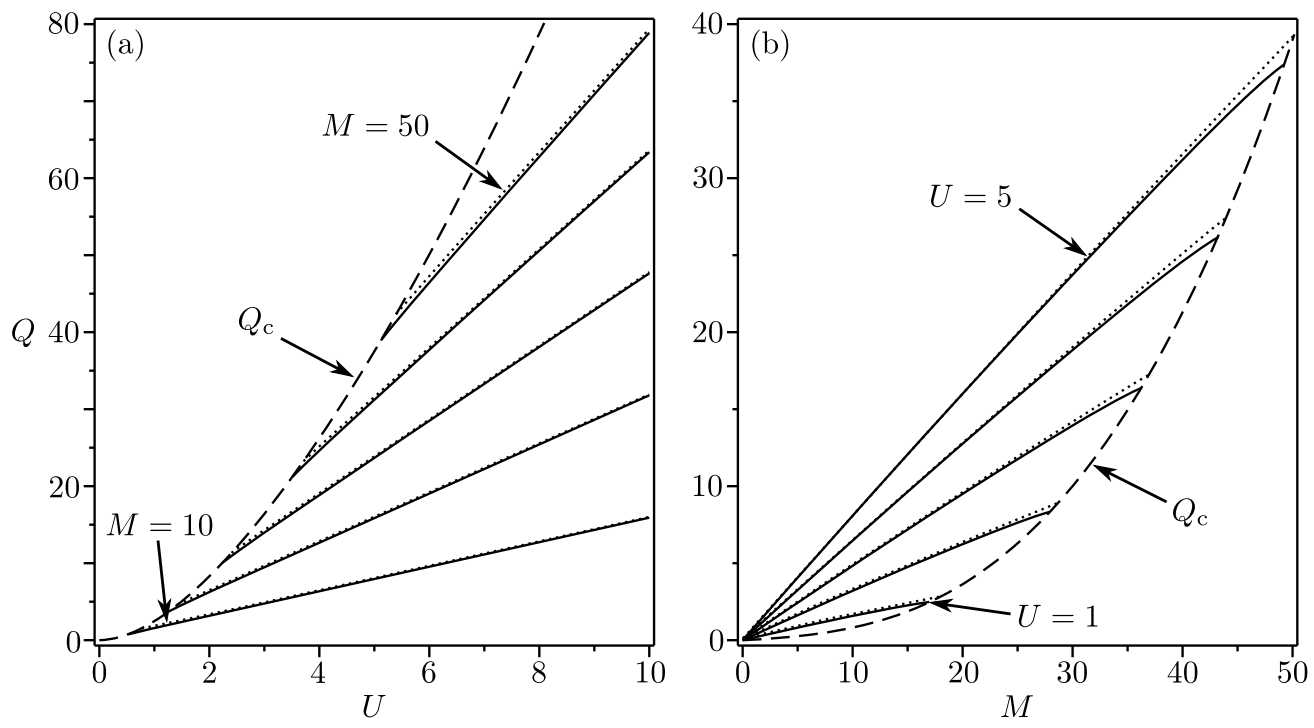

Figure 17. The flux $Q$ plotted as a function of (a) $U$ for $M=10,20,30,40,50$ (solid lines) with the asymptotic solution (4.1) in the limit $U \rightarrow \infty$ (dotted lines) together with the critical flux $Q_{\mathrm{c}}$ (dashed line), and (b) $M$ for $U=1,2,3,4,5$ (solid lines) with the asymptotic solution (4.8) in the limit $M \rightarrow 0$ (dotted lines) together with the critical flux $Q_{\mathrm{c}}$ (dashed line).
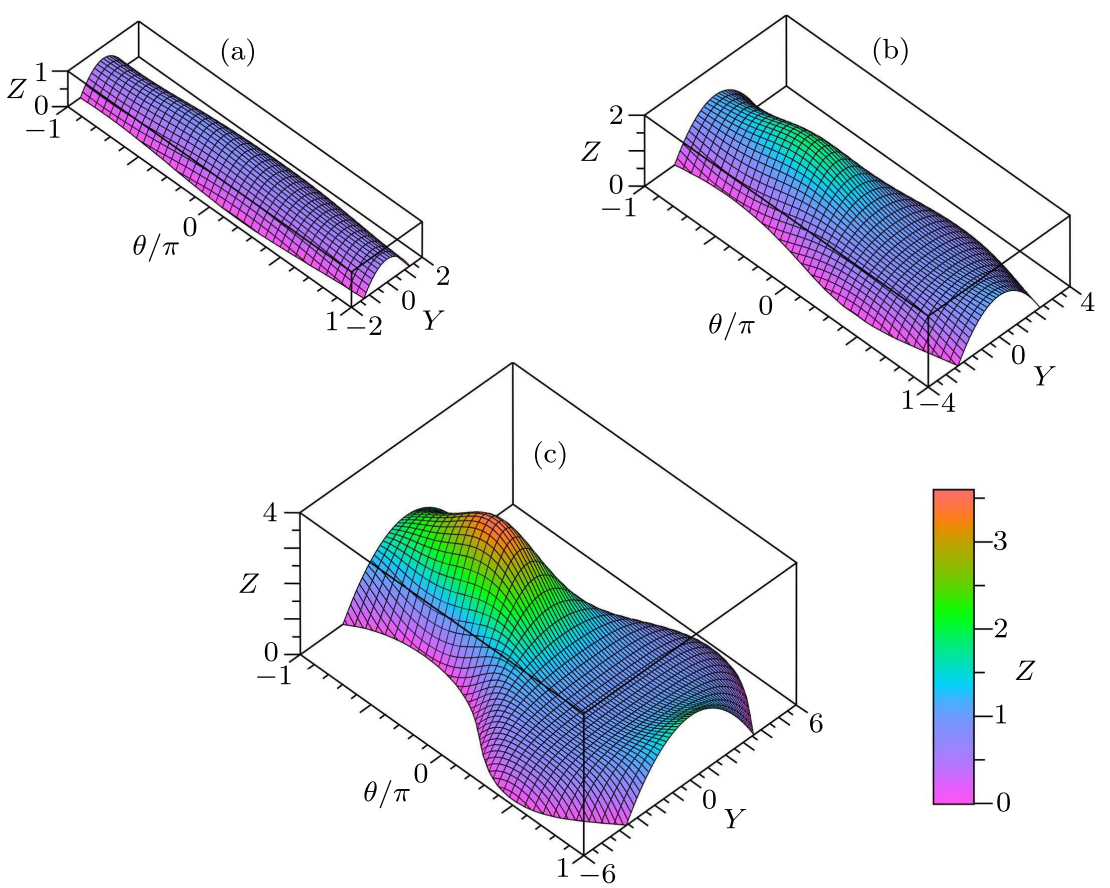

FigURE 18. Three-dimensional plots of the asymptotic solution for the non-critical ring profile $h$ in the limit $U \rightarrow \infty$ as a function of $Y=a y$ and $\theta / \pi$ for (a) $U=1 / 2$ with $M=9$, (b) $U=2$ with $M=27$, and (c) $U=8$ for $M=60$. These plots may be compared with the corresponding exact solutions shown in figures 15(c), 15(f) and 15(i), respectively. 


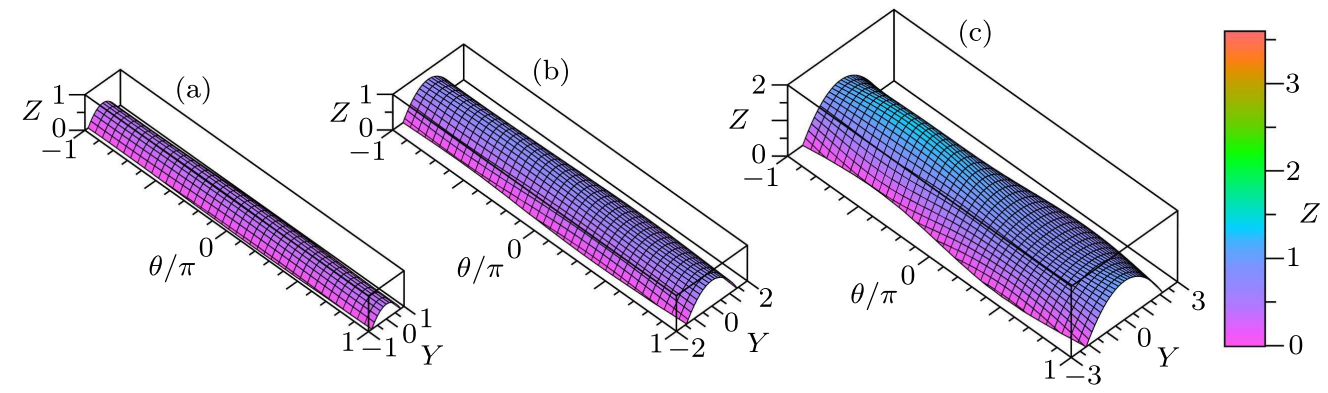

FIgURE 19. Three-dimensional plots of the asymptotic solutions for the non-critical ring profile $h$ in the limit $M \rightarrow 0$ as a function of $Y=a y$ and $\theta / \pi$ for (a) $M=3$ with $U=1 / 2$, (b) $M=9$ with $U=2$, and (c) $M=20$ with $U=8$. These plots may be compared with the corresponding exact solutions shown in figures $15(\mathrm{a}), 15(\mathrm{~d})$ and $15(\mathrm{~g})$, respectively.

\subsection{The limit of small load, $M \rightarrow 0$}

In the limit of small load, $M \rightarrow 0$, with the rotation speed $U$ prescribed, the flux $Q$ and the semi-width $a$ are given from (2.18) and (2.28) by

$$
Q=\frac{M U}{2 \pi}-\frac{9 M^{3}}{9800 \pi^{3} U}+O\left(M^{5}\right)
$$

and

$$
a=\frac{1}{2}\left(\frac{3}{\pi}\right)^{1 / 2}\left[M^{1 / 2}+\frac{(7 U \sin \theta+6 \cos \theta)}{280 \pi U} M^{3 / 2}\right]+O\left(M^{5 / 2}\right) .
$$

Hence the ring profile $h$ is given from (2.10) by

$$
h=\frac{1-y^{2}}{4}\left(\frac{3}{\pi}\right)^{1 / 2}\left[M^{1 / 2}+\frac{\left(7\left(5 y^{2}-3\right) U \sin \theta+12 \cos \theta\right)}{560 \pi U} M^{3 / 2}\right]+O\left(M^{5 / 2}\right) .
$$

The asymptotic solution for the flux $Q$ given by (4.8) shows that the effect of small load is to increase $Q$ from zero at $M=0$. Figure 17(b) also includes the asymptotic solution for $Q$ given by (4.8) shown as dotted lines and shows that, as for the corresponding solution in the limit $U \rightarrow \infty$ described in Subsection 4.1, in practice the asymptotic solution provides a surprisingly good approximation to $Q$ for all $M \leqslant M_{\mathrm{c}}$. Figure 19 shows three-dimensional plots of the asymptotic solution for the non-critical ring profile $h$ for various values of $U$ and $M$, which may be compared with the corresponding exact solutions shown in figures $15(\mathrm{a}), 15(\mathrm{~d})$ and $15(\mathrm{~g})$, respectively. In particular, figure 19 shows that, as for the corresponding solution in the limit $U \rightarrow \infty$ described in Subsection 4.1 , the asymptotic solution for $h$ provides a good approximation to the exact solution for all $M \leqslant M_{\mathrm{c}}$. In particular, the asymptotic solutions for $a$ and $h$ show that the effect of small load is to increase both $a$ and $h$ from zero at $M=0$, and that at leading order in the limit of small load, $M \rightarrow 0$, the ring is narrow and thin with uniform width $a=(3 M / 4 \pi)^{1 / 2} \ll 1$, uniform maximum thickness $h_{\mathrm{m}}=(3 M / 16 \pi)^{1 / 2} \ll 1$, and uniform parabolic profile $h=(3 M / 16 \pi)^{1 / 2}\left(1-y^{2}\right)$.

\section{Occurrence of backflow}

For the two-dimensional full-film flow described by Moffatt (1977), the azimuthal velocity $u$ is always in the same direction as the rotation of the cylinder (i.e. $u \geqslant 0$ for all $-\pi<\theta \leqslant \pi$ and $0 \leqslant z \leqslant 1$ ) and so backflow (i.e. $u<0$ somewhere within the 
flow) never occurs. In contrast, in this Section we will show that for the present threedimensional full-ring flow backflow can occur, but only within a restricted region of the $U-M$ parameter space, and that when it does occur it is always in a small region on the upward-moving side of the cylinder.

From (2.15) the azimuthal velocity may be zero (i.e. $u=0$ ) only on the threedimensional surface defined by

$$
z=1-\left(1-\frac{2 U}{h^{2} \cos \theta}\right)^{1 / 2}
$$

in $0<z \leqslant 1$. It is immediately apparent that (5.1) may have solutions satisfying $z \geqslant 0$ only for $-\pi / 2<\theta<\pi / 2$ (i.e. only on the upward-moving side of the cylinder), and that the surface on which $u=0$ cannot intersect either the substrate $z=0$ (by the no-slip condition) or the stations $\theta= \pm \pi / 2$. If the surface on which $u=0$ intersects the free surface of the fluid $z=1$ then it would do so when $h=(2 U / \cos \theta)^{1 / 2}$, which from $(2.10)$ means that it may intersect the free surface only on the curves $y= \pm y_{\mathrm{s}}(\theta)$ defined by

$$
y_{\mathrm{s}}= \begin{cases}\frac{1}{m a} \cosh ^{-1}\left[\cosh m a-(2 U \tan \theta)^{1 / 2} \sinh m a\right] & \text { if } 0<\theta<\frac{\pi}{2}, \\ \left(1-\frac{2(2 U)^{1 / 2}}{a}\right)^{1 / 2} & \text { if } \theta=0, \\ \frac{1}{m a} \cos ^{-1}\left[\cos m a+(-2 U \tan \theta)^{1 / 2} \sin m a\right] & \text { if }-\frac{\pi}{2}<\theta<0,\end{cases}
$$

which provides an explicit expression for the "footprint" of the region of backflow on the cylinder (i.e. in the $\theta / \pi-Y$ plane). From (2.15) it is clear that at any station $\theta=$ constant the azimuthal velocity $u$ on the free surface $z=1$ has a minimum at $y=0$, since this is where $h$ takes its maximum value $h=h_{\mathrm{m}}$. Hence the endpoints of the region of backflow in the $\theta$ direction (at which, of course, $u=0$ ) will lie on $y=0$, and at these endpoints $h_{\mathrm{m}}=(2 U / \cos \theta)^{1 / 2}$ and $a$ is given by

$$
a= \begin{cases}\frac{2}{m} \tanh ^{-1}(2 U \tan \theta)^{1 / 2} & \text { if } 0<\theta<\frac{\pi}{2}, \\ 2(2 U)^{1 / 2} & \text { if } \theta=0, \\ \frac{2}{m} \tan ^{-1}(-2 U \tan \theta)^{1 / 2} & \text { if }-\frac{\pi}{2}<\theta<0,\end{cases}
$$

so that (2.18) provides an equation determining the values of $\theta$ at the endpoints of any region of backflow in terms of $Q$, namely

$$
Q=\left\{\begin{array}{cc}
-\frac{\cos \theta}{9 m^{4}} F\left(2 \tanh ^{-1}(2 U \tan \theta)^{1 / 2}\right) & \\
+\frac{U}{m^{2}} G\left(2 \tanh ^{-1}(2 U \tan \theta)^{1 / 2}\right) & \text { if } 0<\theta<\frac{\pi}{2}, \\
\frac{304}{105} U^{2} & \text { if } \theta=0, \\
-\frac{\cos \theta}{9 m^{4}} F\left(2 \tan ^{-1}(-2 U \tan \theta)^{1 / 2}\right) & \\
+\frac{U}{m^{2}} G\left(2 \tan ^{-1}(-2 U \tan \theta)^{1 / 2}\right) & \text { if }-\frac{\pi}{2}<\theta<0 .
\end{array}\right.
$$

The function on the right-hand side of (5.4) is real and positive only for $-\pi / 2<\theta<$ 

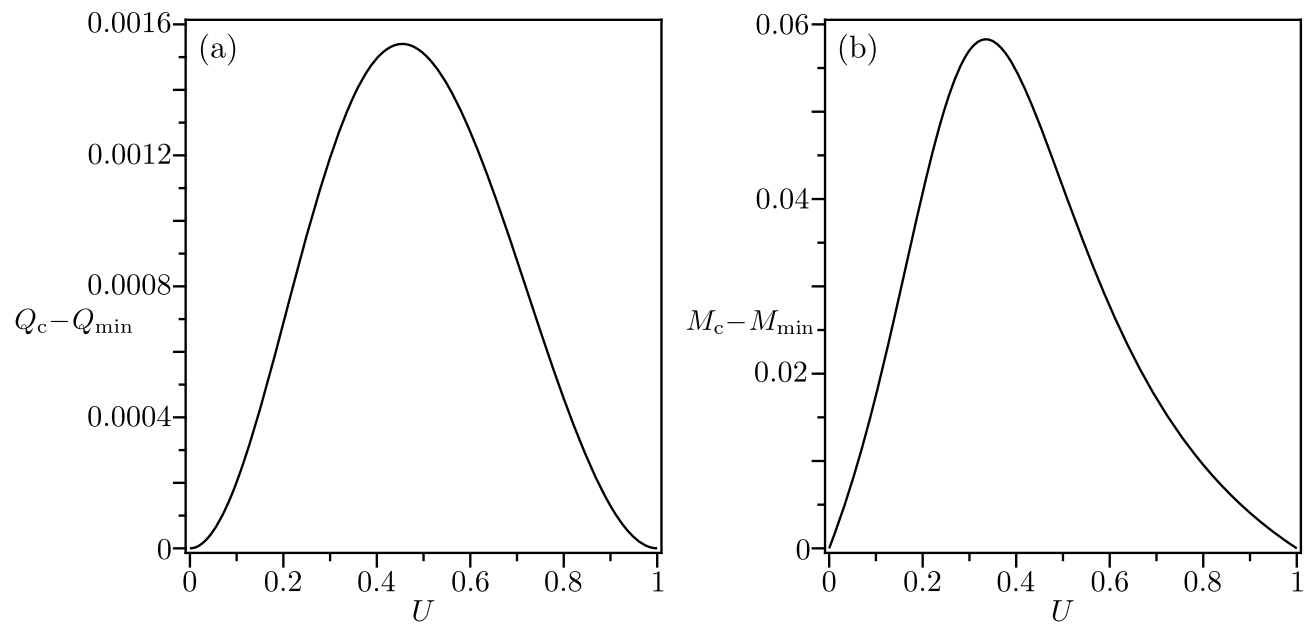

Figure 20. The differences between (a) the critical flux and the minimum flux below which backflow is not possible, $Q_{\mathrm{c}}-Q_{\mathrm{min}}$, and (b) the critical load and the minimum load below which backflow is not possible, $M_{\mathrm{c}}-M_{\mathrm{min}}$, plotted as functions of $U$.

$\theta_{\max }$, where $\theta_{\max }=\theta_{\max }(U)=\cot ^{-1}(2 U)>0$; this function decreases with $\theta$ from infinity as $\theta \rightarrow-\pi / 2^{+}$to a minimum flux denoted by $Q=Q_{\min }$ at some $\theta=\theta_{\min }(U)$ $\left(-\pi / 2<\theta_{\min }<0\right)$, and then increases to infinity as $\theta \rightarrow \theta_{\max }^{-}$. With $U$ prescribed, it can be shown that $Q_{\min }$ satisfies $0<Q_{\min } \leqslant Q_{\mathrm{c}}$ for any $U$ (and there is a corresponding minimum load denoted by $M=M_{\min }$ satisfying $0<M_{\min } \leqslant M_{\mathrm{c}}$ ), and therefore (5.4) has two solutions $\theta=\theta_{1}$ and $\theta=\theta_{2}$ (with $-\pi / 2<\theta_{1}<\theta_{\min }<\theta_{2}<\theta_{\max }$ ) when $Q>Q_{\min }$ (with $Q \leqslant Q_{\mathrm{c}}$ ), one solution $\theta=\theta_{1}=\theta_{2}=\theta_{\min }$ when $Q=Q_{\min }$, and no solution when $Q<Q_{\min }$. However, not all of these solutions for $\theta$ correspond to full-ring solutions; specifically, it is found that for a full-ring solution there may be a region of backflow near the free surface in $\theta_{1} \leqslant \theta \leqslant \theta_{2}$ only when $U<1$ and $Q_{\min }<Q \leqslant Q_{\mathrm{c}}$, and that otherwise the flow is always in the same direction as the rotation of the cylinder. In the special case $Q=Q_{\min }$ the region of backflow collapses to a stagnation point on the free surface at $\theta=\theta_{\min }$. Figure 20 shows the differences between (a) the critical flux and the minimum flux below which backflow is not possible, $Q_{\mathrm{c}}-Q_{\mathrm{min}}$, and (b) the critical load and the minimum load below which backflow is not possible, $M_{\mathrm{c}}-M_{\min }$, plotted as functions of $U$, which, in conjunction with figures 4(b) and 4(c), shows the regions of $U-Q$ and $U-M$ parameter space in which backflow occurs. In particular, figure 20 shows that for a given rotation speed $U$, backflow occurs only when $Q$ and $M$ are very close to $Q_{\mathrm{c}}$ and $M_{\mathrm{c}}$, respectively, and so for brevity we shall henceforth restrict our attention to backflow in the critical case. (The corresponding results in the non-critical case are qualitatively similar, but without the corners that occur in the critical case.)

Figure 21(a) shows the scaled endpoints of the region of backflow in the critical case, $\theta_{1 \mathrm{c}} / \pi$ and $\theta_{2_{\mathrm{c}}} / \pi$, plotted as functions of $U$ together with the scaled position of the corner in the critical solution, $\hat{\theta}_{\mathrm{c}} / \pi$, and, in particular, shows that the endpoints always lie on opposite sides of the corner. It may also be shown that at leading order in the limit $U \rightarrow 0$ the endpoints $\theta_{1 \mathrm{c}}$ and $\theta_{2 \mathrm{c}}$ have the same magnitude but opposite signs, with $\theta_{1 \mathrm{c}}=-\theta_{2 \mathrm{c}}=-\cos ^{-1}(1216 / 1225) \simeq-0.12129(\simeq-0.03861 \pi)$, while at leading order in the limit $U \rightarrow 1^{-}$the endpoints have the same value, with $\theta_{1_{\mathrm{c}}}=\theta_{2_{\mathrm{c}}}=\hat{\theta}_{\mathrm{c}}=-\cot ^{-1} 2 \simeq$ $-0.46365(\simeq-0.14758 \pi)$, showing that the region of backflow has zero width in this case.

Figure 21(b) shows the footprint of the region of backflow in the $\theta / \pi-Y$ plane (i.e. 

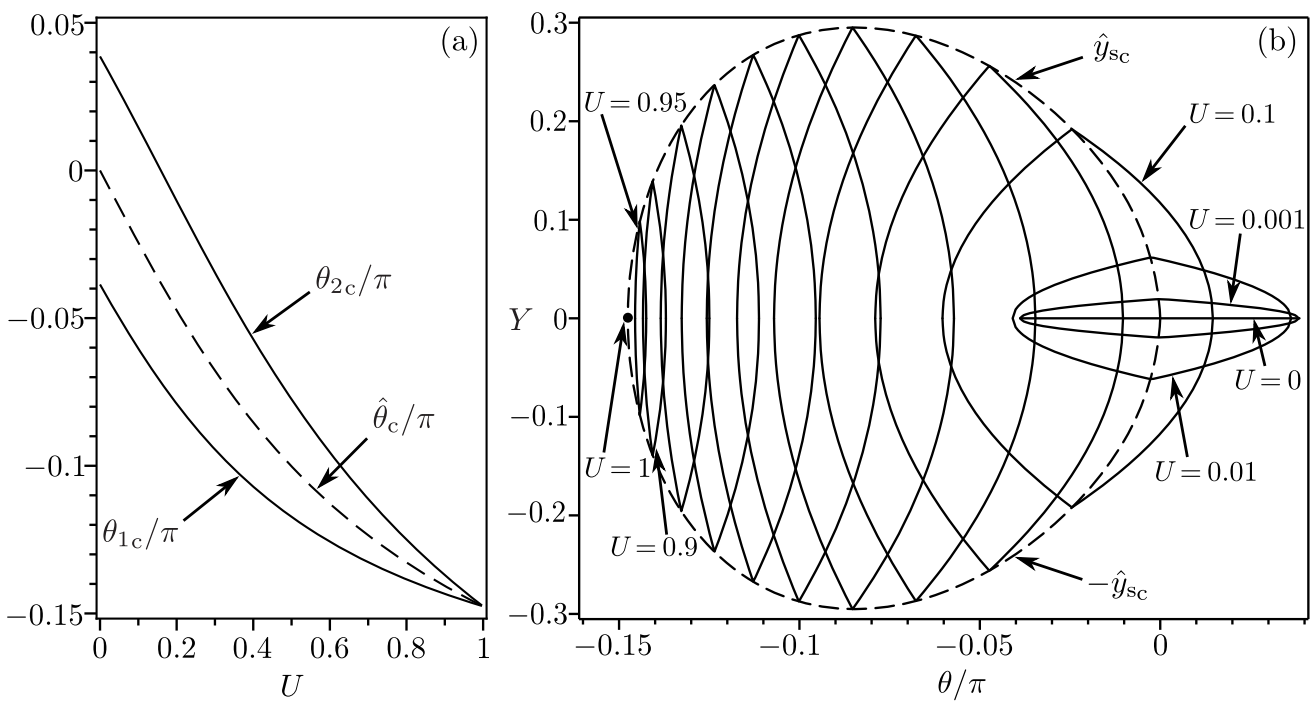

Figure 21. (a) The scaled endpoints of the region of backflow in the critical case, $\theta_{1 \mathrm{c}} / \pi$ and $\theta_{2 \mathrm{c}} / \pi$, plotted as functions of $U$ together with the scaled position of the corner in the critical solution, $\hat{\theta}_{\mathrm{c}} / \pi$. (b) The footprint of the region of backflow in the $\theta / \pi-Y$ plane (i.e. the curves $y= \pm y_{\mathrm{s}}$ given by $\left.(5.2)\right)$ in the critical case for $U=0.1,0.2,0.3, \ldots, 0.9$ and $U=0, U=0.001$, $U=0.01, U=0.95$ (solid lines) and $U=1$ (dot) together with the curves $y= \pm \hat{y}_{\mathrm{s}_{\mathrm{c}}}$, where $\hat{y}_{\mathrm{sc}}=y_{\mathrm{s}}\left(\hat{\theta}_{\mathrm{c}}\right)$, on which the region of backflow has maximum width.

the curves $y= \pm y_{\mathrm{s}}$ given by (5.2)) in the critical case for a range of values of $U$. Figure 21 (b) also shows the curves $y= \pm \hat{y}_{\mathrm{s}_{\mathrm{c}}}$, where $\hat{y}_{\mathrm{s}_{\mathrm{c}}}=y_{\mathrm{s}}\left(\hat{\theta}_{\mathrm{c}}\right)$ (i.e. the value of $y_{\mathrm{s}}$ at the position of the corner $\theta=\hat{\theta}_{c}$ ), on which the region of backflow has maximum width. In particular, figure 21(b) shows that in the limit $U \rightarrow 0$ the region of backflow becomes narrow in the $Y$ direction but approaches a constant width in the $\theta$ direction, eventually becoming a line when $U=0$ (when there is, of course, no full-ring solution), while in the limit $U \rightarrow 1^{-}$it becomes narrow in the $Y$ direction and even narrower in the $\theta$ direction, eventually becoming a point when $U=1$ (representing a stagnation point on the free surface).

\section{Conclusions}

In the present paper we used a combination of asymptotic and numerical methods to investigate the steady three-dimensional flow of a thin, slowly varying ring of Newtonian fluid on either the outside or the inside of a uniformly rotating large horizontal cylinder. Specifically, we studied full-ring solutions, corresponding to a ring of continuous, finite and non-zero thickness that extends all the way around the cylinder. In particular, it is found that, as for the analogous two-dimensional full-film flow described by Moffatt (1977), there is a critical solution corresponding to either a critical load $M=M_{\mathrm{c}}$ above which no full-ring solution exists (if the rotation speed $U$ is prescribed) or a critical rotation speed $U=U_{\mathrm{c}}$ below which no full-ring solution exists (if the load $M$ is prescribed).

In Section 3 we described the behaviour of the critical solution and, in particular, showed that the critical flux $Q_{\mathrm{c}}$, the critical load $M_{\mathrm{c}}$, the critical semi-width $a_{\mathrm{c}}$ and the critical ring profile $h_{\mathrm{c}}$ are all increasing functions of the rotation speed $U$. We also showed that the profile of the critical full-ring solution has a corner analogous to the corner in the critical two-dimensional full-film solution described by Moffatt (1977), but (unlike 
in the the two-dimensional case) that both its position and shape depend on $U$. In the limit of small rotation speed, $U \rightarrow 0$, the critical flux $Q_{\mathrm{c}}=O\left(U^{2}\right) \ll 1$ is small and the critical ring is narrow and thin (with both $a_{\mathrm{c}}$ and $h_{\mathrm{c}}$ being $O\left(U^{1 / 2}\right) \ll 1$ ), leading to a small critical load $M_{\mathrm{c}}=O(U) \ll 1$. In the limit of large rotation speed, $U \rightarrow \infty$, the

critical flux $Q_{\mathrm{c}}=O\left(U^{3 / 2}\right) \gg 1$ is large and the critical ring is wide on the upper half of the cylinder (with $a_{\mathrm{c}}=O\left(U^{1 / 2}\right) \gg 1$ and $h_{\mathrm{m}_{\mathrm{c}}}=O(1)$ ) and thick on the lower half of the cylinder (with $a_{\mathrm{c}}=O(1)$ and $h_{\mathrm{mc}}=O\left(U^{1 / 2}\right) \gg 1$ ), leading to a large critical load $M_{\mathrm{c}}=O\left(U^{1 / 2}\right) \gg 1$. Sketches of the structure of the asymptotic solution in this limit are shown in figure 10.

In Section 4 we described the behaviour of the non-critical full-ring solution which exists only provided that $U>U_{\mathrm{c}}$ and $M<M_{\mathrm{c}}$, where $U_{\mathrm{c}}$ and $M_{\mathrm{c}}$ are the critical values described in Section 3. In particular, we showed that the semi-width $a$ and the ring profile $h$ are increasing functions of $M$ but, in general, non-monotonic functions of $U$, and that while varying $M$ can have a significant effect on the shape of the ring, varying $U$ typically has relatively little effect. In the limit of large rotation speed, $U \rightarrow \infty$, the ring approaches a limiting non-uniform shape (with both $a$ and $h_{\mathrm{m}}$ being $O(1)$ ), whereas in the limit of small load, $M \rightarrow 0$, the ring is narrow and thin (with both $a$ and $h_{\mathrm{m}}$ being $\left.O\left(M^{1 / 2}\right) \ll 1\right)$ with a uniform parabolic profile.

Finally, in Section 5 we showed that, while for most values of $U$ and $M$ the azimuthal velocity is in the same direction as the rotation of the cylinder, unlike in the corresponding two-dimensional full-film flow, there is a region of $U-M$ parameter space close to the critical solution for $U<1$ in which backflow occurs in a small region on the upwardmoving side of the cylinder.

There are several possible worthwhile directions for future work on fluid rings in addition to the piecewise-continuous "shock" or "jump" solutions with one or more discontinuities mentioned in Section 2. In particular, it would be interesting to formulate and solve the evolution equation for the unsteady version of the present steady problem following the approach of, for example, Benilov (2006).

\section{Acknowledgements}

The first author (GAL) gratefully acknowledges the financial support of the United Kingdom Engineering and Physical Sciences Research Council (EPSRC) via a Doctoral Training Account (DTA) research studentship. Part of this work as undertaken while the corresponding author (SKW) was a Visiting Fellow in the Department of Mechanical and Aerospace Engineering, School of Engineering and Applied Science, Princeton University, USA, and part of it was undertaken while he was a Visiting Fellow in the Oxford Centre for Collaborative Applied Mathematics (OCCAM), Mathematical Institute, University of Oxford, United Kingdom. This publication was based on work supported in part by Award No KUK-C1-013-04, made by King Abdullah University of Science and Technology (KAUST).

\section{REFERENCES}

Acrivos, A. \& JIN, B. 2004 Rimming flows within a rotating horizontal cylinder: asymptotic analysis of the thin-film lubrication equations and stability of their solutions. J. Eng. Math. 50, 99-120.

Alekseenko, S. V., Bobylev, A. V. \& Markovich, D. M. 2008 Rivulet flow on the outer surface of an inclined cylinder. J. Eng. Thermophys. 4, 259-272.

Allen, R. F. \& Biggin, C. M. 1974 Longitudinal flow of a lenticular liquid filament down an inclined plane. Phys. Fluids 17, 287-291. 
Ashmore, J., Hosoi, A. E. \& Stone, H. A. 2003 The effect of surface tension on rimming flows in a partially filled rotating cylinder. J. Fluid Mech. 479, 65-98.

Benilov, E. S. 2006 Does surface tension stabilize liquid films inside a rotating horizontal cylinder? Part 2: Multidimensional disturbances. Stud. Appl. Math. 116, 1-20.

Benilov, E. S. 2009 On the stability of shallow rivulets. J. Fluid Mech. 636, 455-474.

Benilov, E. S., Benilov, M. S. \& Kopteva, N. 2008 Steady rimming flows with surface tension. J. Fluid Mech. 597, 91-118.

Benilov, E. S., Benilov, M. S. \& O’Brien, S. G. B. 2009 Existence and stability of regularized shock solutions, with applications to rimming flows. J. Eng. Math. 63, 197-212.

Benilov, E. S., Lapin, V. N. \& O’Brien, S. B. G. 2012 On rimming flows with shocks. J. Eng. Math. 75, 49-62.

Benilov, E. S. \& O'Brien, S. B. G. 2005 Inertial instability of a liquid film inside a rotating horizontal cylinder. Phys. Fluids 17, 052106.

Chen, P.-J., Tsai, Y.-T., Liu, T.-J \& Wu, P.-Y. 2007 Low volume fraction rimming flow in a rotating horizontal cylinder. Phys. Fluids 19, 128107.

Chicharro, R., Vazquez, A. \& Manasseh, R. 2011 Characterization of patterns in rimming flow. Exp. Therm. Fluid Sci. 35, 1184-1192.

Daerr, A., Eggers, J., Limat, L. \& Valade, N. 2011 General mechanism for the meandering instability of rivulets of Newtonian fluids. Phys. Rev. Lett. 106, 184501.

Deans, J. \& Kucuka, S. 2011 The formation of banded condensate films in weak ammoniawater mixtures. J. Heat Transfer 133, 101505.

Diez, J. A., GonzÁlez, A. G. \& Kondic, L. 2009 On the breakup of fluid rivulets. Phys. Fluids 21, 082105.

Duffy, B. R. \& Moffatt, H. K. 1995 Flow of a viscous trickle on a slowly varying incline. Chem. Eng. J. 60, 141-146.

Duffy, B. R. \& Moffatt, H. K. 1997 A similarity solution for viscous source flow on a vertical plane. Eur. J. Appl. Math. 8, 37-47.

Duffy, B. R. \& Wilson, S. K. 1999 Thin-film and curtain flows on the outside of a rotating horizontal cylinder. J. Fluid Mech. 394, 29-49.

Evans, P. L., Schwartz, L. W. \& Roy, R. V. 2004 Steady and unsteady solutions for coating flow on a rotating horizontal cylinder: Two-dimensional theoretical and numerical modeling. Phys. Fluids 16, 2742-2756.

Evans, P. L., Schwartz, L. W. \& Roy, R. V. 2005 Three-dimensional solutions for coating flow on a rotating horizontal cylinder: Theory and experiment. Phys. Fluids 17, 072102.

Hinch, E. J. \& Kelmanson, M. A. 2003 On the decay and drift of free-surface perturbations in viscous thin-film flow exterior to a rotating cylinder. Proc. R. Soc. Lond. A 459, 1193-1213.

Hosoi, A. E. \& Mahadevan, L. 1999 Axial instability of a free-surface front in a partially filled horizontal rotating cylinder. Phys. Fluids 11, 97-106.

Hunt, R. 2008 Numerical solution of the free-surface viscous flow on a horizontal rotating elliptical cylinder. Num. Meth. Part. Diff. Eqns. 24, 1094-1114.

Johnson, R. E. 1988 Steady-state coating flows inside a rotating horizontal cylinder. J. Fluid Mech. 190, 321-342.

Kelmanson, M. A. 2009 On inertial effects in the Moffatt-Pukhnachov coating-flow problem. J. Fluid Mech. 633, 327-353.

KIM, H.-Y., KIM, J.-H. \& KANG, B. H. 2004 Meandering instability of a rivulet. J. Fluid Mech. 498, 245-256.

Le Grand-Piteira, N., Daerr, A. \& Limat, L. 2006 Meandering rivulets on a plane: A simple balance between inertia and capillarity. Phys. Rev. Lett. 96, 254503.

Leslie, G. A., Wilson, S. K. \& Duffy, B. R. 2012 Thermoviscous coating and rimming flow. To appear in Q. J. Mech. Appl. Math.

Melo, F. \& Douady, S. 1993 From solitary waves to static patterns via spatiotemporal intermittency. Phys. Rev. Lett. 71, 3283-3286.

Moffatt, H. K. 1977 Behaviour of a viscous film on the outer surface of a rotating cylinder. J. Méc. 16, 651-673.

NoAkes, C. J., King, J. R. \& Riley, D. S. 2006 On the development of rational approximations incorporating inertial effects in coating and rimming flows: a multiple-scales approach. Q. J. Mech. Appl. Math. 59, 163-190. 
Perazzo, C. A. \& Gratton, J. 2004 Navier-Stokes solutions for parallel flow in rivulets on an inclined plane. J. Fluid Mech. 507, 367-379.

Peterson, R. C., Jimack, P. K. \& Kelmanson, M. A. 2001 On the stability of viscous free-surface flow supported by a rotating cylinder. Proc. R. Soc. Lond. A 457, 1427-1445.

Pougatch, K. \& FrigaArd, I. 2011 Thin film flow on the inside surface of a horizontally rotating cylinder: Steady state solutions and their stability. Phys. Fluids 23, 022102.

Preziosi, L. \& Joseph, D. D. 1988 The run-off condition for coating and rimming flows. $J$. Fluid Mech. 187, 99-113.

Pukhnachev, V. V. 1977 Motion of a liquid film on the surface of a rotating cylinder in a gravitational field. J. Appl. Mech. Tech. Phys. 18, 344-351.

Roy, R. V. \& Schwartz, L. W. 1999 On the stability of liquid ridges. J. Fluid Mech. 391, $293-318$.

SABer, H. H. \& El-Genk, M. S. 2004 On the breakup of a thin liquid film subject to interfacial shear. J. Fluid Mech. 500, 113-133.

Schmuki, P. \& Laso, M. 1990 On the stability of rivulet flow. J. Fluid Mech. 215, 125-143.

Seiden, G. \& Thomas, P. J. 2011 Complexity, segretation, and pattern formation in rotatingdrum flows. Rev. Mod. Phys. 83, 1323-1365.

Shrager, G. R., Shtokolova, M. N. \& Yakutenok, V. A. 2009 Formation of the free surface of a viscous fluid volume inside a rotating horizontal cylinder. Fluid Dynam. 44, 322-327.

Sullivan, J. M., Wilson, S. K. \& Duffy, B. R. 2008 A thin rivulet of perfectly wetting fluid subject to a longitudinal surface shear stress. Q. J. Mech. Appl. Math. 61, 25-61.

Tanasijczuk, A. J., Perazzo, C. A. \& Gratton, J. 2010 Navier-Stokes solutions for steady parallel-sided pendent rivulets. Eur. J. Mech. B. Fluids 29, 465-471.

Thiele, U. 2011 On the depinning of a drop of partially wetting liquid on a rotating cylinder. J. Fluid Mech. 671, 121-136.

Thoroddsen, S. T. \& Mahadevan, L. 1997 Experimental study of coating flows in a partiallyfilled horizontally rotating cylinder. Exp. Fluids 23, 1-13.

Tougher, C. H., Wilson, S. K. \& Duffy, B. R. 2009 On the approach to the critical solution in leading order thin-film coating and rimming flow. Appl. Math. Lett. 22, 882-886.

Towell, G. D. \& Rothfeld, L. B. 1966 Hydrodynamics of rivulet flow. AIChE J. 12, 972-980.

Villegas-Díaz, M., Power, H. \& Riley, D. S. 2005 Analytical and numerical studies of the stability of thin-film rimming flow subject to surface shear. J. Fluid Mech. 541, 317-344.

Williams, J., Hibberd, S., Power, H. \& Riley, D. S. 2012 On the effects of mass and momentum transfer from droplets impacting on steady two-dimensional rimming flow in a horizontal cylinder. Phys. Fluids 24, 053103.

Wilson, S. K. \& Duffy, B. R. 1998 On the gravity-driven draining of a rivulet of viscous fluid down a slowly varying substrate with variation transverse to the direction of flow. Phys. Fluids 10, 13-22.

Wilson, S. K. \& DufFy, B. R. 2005 Unidirectional flow of a thin rivulet on a vertical substrate subject to a prescribed uniform shear stress at its free surface. Phys. Fluids 17, 108105.

Wilson, S. K., Duffy, B. R. \& Hunt, R. 2002 a A slender rivulet of a power-law fluid driven by either gravity or a constant shear stress at the free surface. Q. J. Mech. Appl. Math. 55, 385-408.

Wilson, S. K., Hunt, R. \& Duffy, B. R. $2002 b$ On the critical solutions in coating and rimming flow on a uniformly rotating horizontal cylinder. Q. J. Mech. Appl. Math. 55, $357-383$.

Wilson, S. K., Sullivan, J. M. \& Duffy, B. R. 2011 The energetics of the breakup of a sheet and of a rivulet on a vertical substrate in the presence of a uniform surface shear stress. J. Fluid Mech. 674, 281-306.

Yatim, Y. M., Duffy, B. R., Wilson, S. K. \& Hunt, R. 2011 Similarity solutions for unsteady gravity-driven slender rivulets. Q. J. Mech. Appl. Math. 64, 455-480. 